\title{
DABCO-Catalyzed Reaction of Allenic Esters and Ketones with Salicyl $N$-Tosylimines: Synthesis of Highly Functionalized Chromenes
}

\author{
Yong-Ling Shi and Min Shi* \\ State Key Laboratory of Organometallic Chemistry, \\ Shanghai Institute of Organic Chemistry, \\ Chinese Academy of Sciences, 354 Fenglin Lu, Shanghai 200032 China. \\ Mshi@pub.sioc.ac.cn. Fax: 86-21-64166128.
}

Content

Experimental procedure and Spectroscopic data

X-ray crystal data of $\mathbf{3 a}$ and $\mathbf{4 a}$

S17

NOESY spectra of $\mathbf{1 3 a}$ and $\mathbf{1 3 b}$

S40 


\section{Experimental Procedures}

General Remarks. Unless otherwise stated, all reactions were carried out under argon atmosphere. All solvents were purified by distillation. Other commercially available reagents were used without further purification. Salicyl $N$-tosylimines, ${ }^{1}$ ethyl 2,3-butadienoate, ${ }^{2}$ penta-3,4-dien-2-one ${ }^{3}$ were prepared according to the literature. All reactions were monitored by $\mathrm{TLC}$ with Huanghai $\mathrm{GF}_{254}$ silica gel coated plates. Infrared spectra were measured on a PERKIN-ELMER 983 spectrometer. ${ }^{1} \mathrm{H}$ NMR, ${ }^{13} \mathrm{C}$ NMR and NOESY spectra were recorded on a Varian Mercury vx $300 \mathrm{NMR}$ spectrometer in $\mathrm{CDCl}_{3}$ using tetramethylsilane as the internal standard. Mass spectra were recorded with an HP-5989 instrument and HRMS was measured by an Ion Spec 4.7 Tesla FTMS mass spectrometer. Satisfactory CHN microanalyses were obtained with a Carlo-Erba 1106 analyzer. Melting points were obtained by means of a micro melting point apparatus and are uncorrected.

\section{Characterization Data for the New Salicyl $N$-Tosylimines are Presented as Below.}

$N$-(2-Hydroxy-3-methoxybenzylidene)-4-methylbenzenesulfonamide: a yellow solid: mp. 107-109 ${ }^{\circ} \mathrm{C}$; IR $\left(\mathrm{CH}_{2} \mathrm{Cl}_{2}\right) \vee 3396,1596,1567,1257,1159,1089 \mathrm{~cm}^{-1} ;{ }^{1} \mathrm{H}$ NMR $\left(\mathrm{CDCl}_{3}, \mathrm{TMS}\right.$, $300 \mathrm{MHz}) \delta 2.44$ (3H, s, Me), 3.90 (3H, s, MeO), 6.92-6.97 (1H, m, Ar), 7.08-7.16 (2H, m, Ar), $7.34(2 \mathrm{H}, \mathrm{d}, J=8.1 \mathrm{~Hz}, \mathrm{Ar}), 7.86(2 \mathrm{H}, \mathrm{d}, J=8.1 \mathrm{~Hz}, \mathrm{Ar}), 9.12(1 \mathrm{H}, \mathrm{s}, \mathrm{OH}), 10.74(1 \mathrm{H}, \mathrm{s}, \mathrm{CH})$.

${ }^{13} \mathrm{C} \mathrm{NMR}\left(\mathrm{CDCl}_{3}, \mathrm{TMS}, 75.44 \mathrm{MHz}\right) \delta 21.5,56.2,116.6,118.0,119.8,125.6,127.7,129.8$, 134.8, 144.9, 148.2, 151.9, 171.0. MS (EI) m/e $305\left(\mathrm{M}^{+}, 84.0\right), 150$ ( $\left.\mathrm{M}^{+}-155,100\right)$. Anal. Calcd. for $\mathrm{C}_{15} \mathrm{H}_{15} \mathrm{NO}_{4} \mathrm{~S}$ requires C, 59.00; H, 4.95; N, 4.59\%. Found: C, 58.93; H, 4.94; N, $4.56 \%$. 
$N$-(2-Hydroxy-4-methoxybenzylidene)-4-methylbenzenesulfonamide: a colorless solid: mp. $115-117{ }^{\circ} \mathrm{C}$; IR $\left(\mathrm{CH}_{2} \mathrm{Cl}_{2}\right)$ v 3067, 1632, 1586, 1504, 1158, $1087 \mathrm{~cm}^{-1} ;{ }^{1} \mathrm{H} \mathrm{NMR}\left(\mathrm{CDCl}_{3}\right.$, TMS, $300 \mathrm{MHz}) \delta 2.44(3 \mathrm{H}, \mathrm{s}, \mathrm{Me}), 3.86(3 \mathrm{H}, \mathrm{s}, \mathrm{MeO}), 6.44(1 \mathrm{H}, \mathrm{d}, J=2.4 \mathrm{~Hz}, \mathrm{Ar}), 6.56(1 \mathrm{H}$, $\mathrm{dd}, J=8.7,2.4 \mathrm{~Hz}, \mathrm{Ar}), 7.32-7.39(3 \mathrm{H}, \mathrm{m}, \mathrm{Ar}), 7.84(2 \mathrm{H}, \mathrm{d}, J=8.7 \mathrm{~Hz}, \mathrm{Ar}), 8.96(1 \mathrm{H}, \mathrm{s}, \mathrm{OH})$, $11.21(1 \mathrm{H}, \mathrm{s}, \mathrm{CH}) .{ }^{13} \mathrm{C} \mathrm{NMR}\left(\mathrm{CDCl}_{3}, \mathrm{TMS}, 75.44 \mathrm{MHz}\right) \delta 21.6,55.8,100.9,109.4,110.6$, 127.6, 129.9, 135.7, 136.9, 144.6, 164.9, 167.4, 169.9. MS (EI) m/e $305\left(\mathrm{M}^{+}, 79.0\right), 91$ $\left(\mathrm{M}^{+}-206,100\right)$. Anal. Calcd. for $\mathrm{C}_{15} \mathrm{H}_{15} \mathrm{NO}_{4} \mathrm{~S}$ requires $\mathrm{C}, 59.00 ; \mathrm{H}, 4.95 ; \mathrm{N}, 4.59 \%$. Found: $\mathrm{C}$, $58.86 ; \mathrm{H}, 4.98 ; \mathrm{N}, 4.59 \%$.

$N$-(2-Hydroxy-5-methoxybenzylidene)-4-methylbenzenesulfonamide: a yellow solid: mp. $158-159{ }^{\circ} \mathrm{C} ; \mathrm{IR}\left(\mathrm{CH}_{2} \mathrm{Cl}_{2}\right) \vee 3067,2951,1562,1487,1275,1160 \mathrm{~cm}^{-1} ;{ }^{1} \mathrm{H} \mathrm{NMR}\left(\mathrm{CDCl}_{3}, \mathrm{TMS}\right.$, $300 \mathrm{MHz}) \delta 2.44$ (3H, s, Me), 3.79 (3H, s, MeO), 6.92-6.95 (2H, m, Ar), 7.12-7.16 (1H, m, Ar), $7.35(2 \mathrm{H}, \mathrm{d}, J=8.4 \mathrm{~Hz}, \mathrm{Ar}), 7.86(2 \mathrm{H}, \mathrm{d}, J=8.4 \mathrm{~Hz}, \mathrm{Ar}), 9.06(1 \mathrm{H}, \mathrm{s}, \mathrm{OH}), 10.49(1 \mathrm{H}, \mathrm{s}, \mathrm{CH})$. ${ }^{13} \mathrm{C} \mathrm{NMR}\left(\mathrm{CDCl}_{3}, \mathrm{TMS}, 75.44 \mathrm{MHz}\right) \delta 21.6,55.8,115.9,116.1,118.9,125.9,127.8,130.0$, 135.0, 145.0, 152.8, 156.7, 171.2. MS (EI) m/e $305\left(\mathrm{M}^{+}, 63.8\right), 150\left(\mathrm{M}^{+}-155,100\right)$. Anal. Calcd. for $\mathrm{C}_{15} \mathrm{H}_{15} \mathrm{NO}_{4} \mathrm{~S}$ requires $\mathrm{C}, 59.00 ; \mathrm{H}, 4.95 ; \mathrm{N}, 4.59 \%$. Found: C, 58.90; H, 5.02; N, $4.44 \%$.

$N$-(2-Hydroxy-5-methylbenzylidene)-4-methylbenzenesulfonamide: a yellow solid: mp. $188-190{ }^{\circ} \mathrm{C} ; \mathrm{IR}\left(\mathrm{CH}_{2} \mathrm{Cl}_{2}\right) \vee 3074,1596,1486,1324,1285,1159 \mathrm{~cm}^{-1} ;{ }^{1} \mathrm{H} \mathrm{NMR}\left(\mathrm{CDCl}_{3}, \mathrm{TMS}\right.$, $300 \mathrm{MHz}) \delta 2.31(3 \mathrm{H}, \mathrm{s}, \mathrm{Me}), 2.44(3 \mathrm{H}, \mathrm{s}, \mathrm{Me}), 6.90(1 \mathrm{H}, \mathrm{d}, J=8.7 \mathrm{~Hz}, \mathrm{Ar}), 7.26-7.36(4 \mathrm{H}, \mathrm{m}$, 
Ar), $7.85(2 \mathrm{H}, \mathrm{d}, J=8.4 \mathrm{~Hz}, \mathrm{Ar}), 9.04(1 \mathrm{H}, \mathrm{s}, \mathrm{OH}), 10.64(1 \mathrm{H}, \mathrm{s}, \mathrm{CH}) .{ }^{13} \mathrm{C} \mathrm{NMR}\left(\mathrm{CDCl}_{3}, \mathrm{TMS}\right.$, $75.44 \mathrm{MHz}) \delta 20.1,21.6,116.2,117.6,127.8,129.5,129.9,134.9,135.0,138.5,144.9,160.0$, 171.4. MS (EI) m/e $289\left(\mathrm{M}^{+}, 96.0\right), 91\left(\mathrm{M}^{+}-191,100\right)$. Anal. Calcd. for $\mathrm{C}_{15} \mathrm{H}_{15} \mathrm{NO}_{3} \mathrm{~S}$ requires C, 62.26; H, 5.23; N, 4.84\%. Found: C, 62.21; H, 5.20; N, 4.64\%.

$N$-(5-Bromo-2-hydroxybenzylidene)-4-methylbenzenesulfonamide: a yellow solid: $\mathrm{mp}$. $183-185{ }^{\circ} \mathrm{C}$; IR $\left(\mathrm{CH}_{2} \mathrm{Cl}_{2}\right) \vee 3052,1594,1472,1337,1280,1159 \mathrm{~cm}^{-1} ;{ }^{1} \mathrm{H}$ NMR $\left(\mathrm{CDCl}_{3}, \mathrm{TMS}\right.$, $300 \mathrm{MHz}) \delta 2.46(3 \mathrm{H}, \mathrm{s}, \mathrm{Me}), 6.91(1 \mathrm{H}, \mathrm{d}, J=8.7 \mathrm{~Hz}, \mathrm{Ar}), 7.37(2 \mathrm{H}, \mathrm{d}, J=8.4 \mathrm{~Hz}, \mathrm{Ar})$, 7.56-7.62 (2H, m, Ar), $7.86(2 \mathrm{H}, \mathrm{d}, J=8.4 \mathrm{~Hz}, \mathrm{Ar}), 9.01(1 \mathrm{H}, \mathrm{s}, \mathrm{OH}), 10.82(1 \mathrm{H}, \mathrm{s}, \mathrm{CH}) .{ }^{13} \mathrm{C}$ NMR $\left(\mathrm{CDCl}_{3}\right.$, TMS, 75.44 MHz) $\delta 21.7,111.6,117.8,119.8,128.0,130.0,134.4,136.8$, 139.6, 145.3, 160.9, 170.1. MS (EI) m/e $355\left(\mathrm{M}^{+}+2,26.4\right), 353\left(\mathrm{M}^{+}, 25.7\right), 91\left(\mathrm{M}^{+}-262,100\right)$. Anal. Calcd. for $\mathrm{C}_{14} \mathrm{H}_{12} \mathrm{BrNO}_{3} \mathrm{~S}$ requires $\mathrm{C}, 47.47 ; \mathrm{H}, 3.41 ; \mathrm{N}, 3.95 \%$. Found: $\mathrm{C}, 47.41 ; \mathrm{H}$, $3.33 ; \mathrm{N}, 3.93 \%$.

$N$-(3,5-Dichloro-2-hydroxybenzylidene)-4-methylbenzenesulfonamide: a yellow solid: mp. $183-184{ }^{\circ} \mathrm{C} ; \mathrm{IR}\left(\mathrm{CH}_{2} \mathrm{Cl}_{2}\right) \vee 3071,1594,1557,1334,1294,1161 \mathrm{~cm}^{-1} ;{ }^{1} \mathrm{H}$ NMR $\left(\mathrm{CDCl}_{3}, \mathrm{TMS}\right.$, $300 \mathrm{MHz}) \delta 2.46(3 \mathrm{H}, \mathrm{s}, \mathrm{Me}), 7.37(2 \mathrm{H}, \mathrm{d}, J=8.1 \mathrm{~Hz}, \mathrm{Ar}), 7.44(1 \mathrm{H}, \mathrm{d}, J=2.4 \mathrm{~Hz}, \mathrm{Ar}), 7.58$ $(1 \mathrm{H}, \mathrm{d}, J=2.4 \mathrm{~Hz}, \mathrm{Ar}), 7.86(2 \mathrm{H}, \mathrm{d}, J=8.1 \mathrm{~Hz}, \mathrm{Ar}), 9.03(1 \mathrm{H}, \mathrm{s}, \mathrm{OH}), 11.35(1 \mathrm{H}, \mathrm{s}, \mathrm{CH}) .{ }^{13} \mathrm{C}$ NMR $\left(\mathrm{CDCl}_{3}, \mathrm{TMS}, 75.44 \mathrm{MHz}\right) \delta 21.7,117.8,123.6,124.8,128.1,130.1,132.2,134.1$, 136.2, 145.6, 156.1, 169.5. MS (EI) m/e $343\left(\mathrm{M}^{+}, 31.2\right), 91\left(\mathrm{M}^{+}-252,100\right)$. Anal. Calcd. for $\mathrm{C}_{14} \mathrm{H}_{11} \mathrm{NO}_{3} \mathrm{Cl}_{2} \mathrm{~S}$ requires $\mathrm{C}, 48.85 ; \mathrm{H}, 3.22 ; \mathrm{N}, 4.07 \%$. Found: C, 48.86; H, 3.28; $\mathrm{N}, 4.04 \%$. 
Typical Reaction Procedure for $\mathrm{PPh}_{2} \mathrm{Me}$-catalyzed Reaction of Ethyl 2,3-butadienoate 2a with Salicyl $N$-tosylimine $1 a$.

To a solution of salicyl $N$-tosylimine $\mathbf{1 a}(138 \mathrm{mg}, 0.50 \mathrm{mmol})$ and ethyl 2,3-butadienoate $\mathbf{2 a}$ $(69 \mu \mathrm{L}, 0.60 \mathrm{mmol})$ in THF $(2.0 \mathrm{~mL})$ at room temperature was added $\mathrm{PPh}_{2} \mathrm{Me}(23 \mu \mathrm{L}, 0.0625$ mmol) and the reaction mixture was further stirred at room temperature. The reaction was monitored by TLC plate. When 1a disappeared, the solvent was removed under reduced pressure and the residue was purified by a flash chromatography (eluent: EtOAc:Petroleum ether $=1: 4)$ to yield $\mathbf{3 a}(159 \mathrm{mg}, 82 \%)$ as a colorless solid.

2-(2-Hydroxy-phenyl)-1-(toluene-4-sulfonyl)-2,5-dihydro-1H-pyrrole-3-carboxylic acid ethyl ester (3a): a colorless solid: mp. 156-158 ${ }^{\circ} \mathrm{C}$; IR $\left(\mathrm{CH}_{2} \mathrm{Cl}_{2}\right) \vee 3427,1718,1344,1258$, 1162, $1094 \mathrm{~cm}^{-1} ;{ }^{1} \mathrm{H} \mathrm{NMR}\left(\mathrm{CDCl}_{3}, \mathrm{TMS}, 300 \mathrm{MHz}\right) \delta 1.12\left(3 \mathrm{H}, \mathrm{t}, J=7.2 \mathrm{~Hz}, \mathrm{CH}_{3}\right), 2.38(3 \mathrm{H}$, s, Me), 3.98-4.16 (2H, m, $\left.\mathrm{CH}_{2}\right), 4.35-4.53\left(2 \mathrm{H}, \mathrm{m}, \mathrm{CH}_{2}\right), 6.07(1 \mathrm{H}, \mathrm{t}, J=2.7 \mathrm{~Hz}), 6.77-6.91$

(4H, m, Ar), 7.00-7.03 (1H, m, Ar), 7.12-7.22 (3H, m, Ar), $7.56(2 \mathrm{H}, \mathrm{d}, J=8.4 \mathrm{~Hz}, \mathrm{Ar}) .{ }^{13} \mathrm{C}$ NMR $\left(\mathrm{CDCl}_{3}, \mathrm{TMS}, 75.44 \mathrm{MHz}\right) \delta 13.8,21.5,54.9,61.3,63.2,118.3,121.1,126.6,127.3$, 128.0, 129.3, 129.6, 134.5, 135.1, 136.3, 143.7, 153.8, 162.6. MS (EI) m/e $387\left(\mathrm{M}^{+}, 28.3\right), 91$ $\left(\mathrm{M}^{+}-296,100\right)$. Anal. Calcd. for $\mathrm{C}_{20} \mathrm{H}_{21} \mathrm{NO}_{5} \mathrm{~S}$ requires $\mathrm{C}, 62.00 ; \mathrm{H}, 5.46 ; \mathrm{N}, 3.62 \%$. Found: $\mathrm{C}$, $62.08 ; \mathrm{H}, 5.38 ; \mathrm{N}, 3.54 \%$.

Typical Reaction Procedure for DABCO-catalyzed Reaction of Ethyl 2,3-butadienoate 2a with Salicyl $N$-tosylimine $1 a$.

To a flame-dried Schlenk tube at room temperature was in turn added molecular sieve 4A (100 
mg), DABCO (5.6 mg, $0.05 \mathrm{mmol})$, salicyl $N$-tosylimine $1 \mathrm{a}(138 \mathrm{mg}, 0.50 \mathrm{mmol})$, THF (2.0 $\mathrm{mL})$ and ethyl 2,3-butadienoate $\mathbf{2 a}(69 \mu \mathrm{L}, 0.60 \mathrm{mmol})$ and the reaction mixture was further stirred at room temperature. The reaction was monitored by TLC plate. When 1a disappeared, the solvent was removed under reduced pressure and the residue was purified by a flash chromatography (eluent: EtOAc:Petroleum ether = 1:4-2:1) to yield 4a (169 mg, $87 \%)$ as a colorless solid.

2-Methyl-4-(toluene-4-sulfonylamino)-4H-chromene-3-carboxylic acid ethyl ester (4a): a colorless solid: mp. $130-132{ }^{\circ} \mathrm{C}$; IR $\left(\mathrm{CH}_{2} \mathrm{Cl}_{2}\right) \vee 3273,1710,1638,1347,1223,1157 \mathrm{~cm}^{-1} ;{ }^{1} \mathrm{H}$ NMR ( $\left.\mathrm{CDCl}_{3}, \mathrm{TMS}, 300 \mathrm{MHz}\right) \delta 1.22\left(3 \mathrm{H}, \mathrm{t}, J=7.2 \mathrm{~Hz}, \mathrm{CH}_{3}\right), 2.39(3 \mathrm{H}, \mathrm{s}, \mathrm{Me}), 2.40(3 \mathrm{H}, \mathrm{s}$, Me), $3.90(1 \mathrm{H}, \mathrm{dq}, J=10.8,7.2 \mathrm{~Hz}), 4.10(1 \mathrm{H}, \mathrm{dq}, J=10.8,7.2 \mathrm{~Hz}), 5.00(1 \mathrm{H}, \mathrm{d}, J=6.6 \mathrm{~Hz}$, NH), $5.60(1 \mathrm{H}, \mathrm{d}, J=6.6 \mathrm{~Hz}, \mathrm{CH}), 7.00-7.05(2 \mathrm{H}, \mathrm{m}, \mathrm{Ar}), 7.18-7.28(3 \mathrm{H}, \mathrm{m}, \mathrm{Ar}), 7.34-7.36$ $(1 \mathrm{H}, \mathrm{m}, \mathrm{Ar}), 7.55(2 \mathrm{H}, \mathrm{d}, J=8.1 \mathrm{~Hz}, \mathrm{Ar}) .{ }^{13} \mathrm{C} \mathrm{NMR}\left(\mathrm{CDCl}_{3}, \mathrm{TMS}, 75.44 \mathrm{MHz}\right) \delta 14.1,19.7$, $21.4,47.8,60.5,102.9,116.1,120.0,124.6,126.7,129.0,129.1,129.6,139.2,142.6,150.5$, 163.1, 166.3. MS (EI) m/e $232\left(\mathrm{M}^{+}-155,39.7\right), 217\left(\mathrm{M}^{+}-170,100\right)$. Anal. Calcd. for $\mathrm{C}_{20} \mathrm{H}_{21} \mathrm{NO}_{5} \mathrm{~S}$ requires C, 62.00; H, 5.46; N, 3.62\%. Found: C, 61.73; H, 5.52; N, 3.55\%.

6-Methoxy-2-methyl-4-(toluene-4-sulfonylamino)-4H-chromene-3-carboxylic acid ethyl ester (4b): a colorless solid: mp. $150-151{ }^{\circ} \mathrm{C}$; IR $\left(\mathrm{CH}_{2} \mathrm{Cl}_{2}\right) \vee 3275,1710,1498,1218,1156$, $1070 \mathrm{~cm}^{-1} ;{ }^{1} \mathrm{H} \mathrm{NMR}\left(\mathrm{CDCl}_{3}\right.$, TMS, $\left.300 \mathrm{MHz}\right) \delta 1.23\left(3 \mathrm{H}, \mathrm{t}, J=7.2 \mathrm{~Hz}, \mathrm{CH}_{3}\right), 2.39(6 \mathrm{H}, \mathrm{s}$, 2Me), $3.65(3 \mathrm{H}, \mathrm{s}, \mathrm{MeO}), 3.92(1 \mathrm{H}, \mathrm{dq}, J=10.5,7.2 \mathrm{~Hz}), 4.12(1 \mathrm{H}, \mathrm{dq}, J=10.5,7.2 \mathrm{~Hz}), 5.05$ $(1 \mathrm{H}, \mathrm{d}, J=6.3 \mathrm{~Hz}, \mathrm{NH}), 5.56(1 \mathrm{H}, \mathrm{d}, J=6.3 \mathrm{~Hz}, \mathrm{CH}), 6.74(1 \mathrm{H}, \mathrm{d}, J=2.7 \mathrm{~Hz}, \mathrm{Ar}), 6.79(1 \mathrm{H}$, 
$\mathrm{dd}, J=8.7,2.7 \mathrm{~Hz}, \mathrm{Ar}), 6.97(1 \mathrm{H}, \mathrm{d}, J=8.7 \mathrm{~Hz}, \mathrm{Ar}), 7.18(2 \mathrm{H}, \mathrm{d}, J=7.8 \mathrm{~Hz}, \mathrm{Ar}), 7.53(2 \mathrm{H}, \mathrm{d}$, $J=7.8 \mathrm{~Hz}, \mathrm{Ar}) .{ }^{13} \mathrm{C} \mathrm{NMR}\left(\mathrm{CDCl}_{3}\right.$, TMS, $\left.75.44 \mathrm{MHz}\right) \delta 14.1,19.8,21.4,48.4,55.3,60.5$, 102.0, 111.9, 116.5, 117.2, 120.1, 126.6, 129.1, 139.3, 142.6, 144.7, 156.1, 163.3, 166.5. MS (EI) m/e $417\left(\mathrm{M}^{+}, 3.3\right), 247\left(\mathrm{M}^{+}-170,100\right)$. Anal. Calcd. for $\mathrm{C}_{21} \mathrm{H}_{23} \mathrm{NO}_{6} \mathrm{~S}$ requires $\mathrm{C}, 60.42, \mathrm{H}$, 5.55; N, 3.36\%. Found: C, 60.31; H, 5.34; N, 3.31\%.

8-Methoxy-2-methyl-4-(toluene-4-sulfonylamino)-4H-chromene-3-carboxylic acid ethyl ester (4c): a white solid: mp. 163-164 ${ }^{\circ} \mathrm{C}$; IR $\left(\mathrm{CH}_{2} \mathrm{Cl}_{2}\right) \vee 3305,1705,1329,1212,1155,1098$ $\mathrm{cm}^{-1} ;{ }^{1} \mathrm{H} \mathrm{NMR}\left(\mathrm{CDCl}_{3}, \mathrm{TMS}, 300 \mathrm{MHz}\right) \delta 1.22\left(3 \mathrm{H}, \mathrm{t}, J=7.2 \mathrm{~Hz}, \mathrm{CH}_{3}\right), 2.35(3 \mathrm{H}, \mathrm{s}, \mathrm{Me})$, $2.39(3 \mathrm{H}, \mathrm{s}, \mathrm{Me}), 3.88(3 \mathrm{H}, \mathrm{s}, \mathrm{MeO}), 3.89(1 \mathrm{H}, \mathrm{dq}, J=10.5,7.2 \mathrm{~Hz}), 4.10(1 \mathrm{H}, \mathrm{dq}, J=10.5$, $7.2 \mathrm{~Hz}), 5.03(1 \mathrm{H}, \mathrm{d}, J=6.6 \mathrm{~Hz}, \mathrm{NH}), 5.64(1 \mathrm{H}, \mathrm{d}, J=6.6 \mathrm{~Hz}, \mathrm{CH}), 6.80-6.83(1 \mathrm{H}, \mathrm{m}, \mathrm{Ar})$, 6.98-7.00 (2H, m, Ar), 7.21 (2H, d, $J=7.8 \mathrm{~Hz}, \mathrm{Ar}), 7.61(2 \mathrm{H}, \mathrm{d}, J=7.8 \mathrm{~Hz}, \mathrm{Ar}) .{ }^{13} \mathrm{C}$ NMR $\left(\mathrm{CDCl}_{3}, \mathrm{TMS}, 75.44 \mathrm{MHz}\right) \delta 14.1,19.3,21.4,47.5,55.9,60.5,103.0,110.8,120.8,121.5$, 124.5, 126.7, 129.1, 139.4, 140.1, 142.6, 147.1, 162.7, 166.3. MS (EI) m/e $417\left(\mathrm{M}^{+}, 1.5\right), 247$ $\left(\mathrm{M}^{+}-170,100\right)$. Anal. Calcd. for $\mathrm{C}_{21} \mathrm{H}_{23} \mathrm{NO}_{6} \mathrm{~S}$ requires $\mathrm{C}, 60.42, \mathrm{H}, 5.55 ; \mathrm{N}, 3.36 \%$. Found: $\mathrm{C}$, $60.62 ; \mathrm{H}, 5.56 ; \mathrm{N}, 3.36 \%$.

2,6-Dimethyl-4-(toluene-4-sulfonylamino)-4H-chromene-3-carboxylic acid ethyl ester (4d): a white solid: mp. $118-120{ }^{\circ} \mathrm{C}$; IR $\left(\mathrm{CH}_{2} \mathrm{Cl}_{2}\right) \vee 3275,1709,1499,1339,1219,1157 \mathrm{~cm}^{-1}$; ${ }^{1} \mathrm{H}$ NMR $\left(\mathrm{CDCl}_{3}\right.$, TMS, $\left.300 \mathrm{MHz}\right) \delta 1.25\left(3 \mathrm{H}, \mathrm{t}, J=6.9 \mathrm{~Hz}, \mathrm{CH}_{3}\right), 2.14(3 \mathrm{H}, \mathrm{s}, \mathrm{Me}), 2.39(6 \mathrm{H}$, s, 2Me), $3.95(1 \mathrm{H}, \mathrm{dq}, J=11.4,6.9 \mathrm{~Hz}), 4.12(1 \mathrm{H}, \mathrm{dq}, J=11.4,6.9 \mathrm{~Hz}), 5.07(1 \mathrm{H}, \mathrm{d}, J=6.6$ $\mathrm{Hz}, \mathrm{NH}), 5.52(1 \mathrm{H}, \mathrm{d}, J=6.6 \mathrm{~Hz}, \mathrm{CH}), 6.90-7.03(3 \mathrm{H}, \mathrm{m}, \mathrm{Ar}), 7.18(2 \mathrm{H}, \mathrm{d}, J=8.1 \mathrm{~Hz}, \mathrm{Ar})$, 
$7.51(2 \mathrm{H}, \mathrm{d}, J=8.1 \mathrm{~Hz}, \mathrm{Ar}) .{ }^{13} \mathrm{C} \mathrm{NMR}\left(\mathrm{CDCl}_{3}, \mathrm{TMS}, 75.44 \mathrm{MHz}\right) \delta 14.1,19.8,20.5,21.4$, $48.0,60.5,102.8,115.9,119.1,126.7,129.0,129.6,129.7,134.1,139.3,142.5,148.6,163.2$ 166.5. MS (EI) m/e $401\left(\mathrm{M}^{+}, 1.4\right), 231\left(\mathrm{M}^{+}-170,100\right)$. Anal. Calcd. for $\mathrm{C}_{21} \mathrm{H}_{23} \mathrm{NO}_{5} \mathrm{~S}$ requires C, 62.82, H, 5.77; N, 3.49\%. Found: C, 62.92; H, 5.85; N, 3.34\%.

6,8-Dichloro-2-methyl-4-(toluene-4-sulfonylamino)-4H-chromene-3-carboxylic acid ethyl ester (4e): a white solid: $\mathrm{mp} .141-142{ }^{\circ} \mathrm{C}$; IR $\left(\mathrm{CH}_{2} \mathrm{Cl}_{2}\right)$ v 3272, 1716, 1463, 1245, 1201, $1158 \mathrm{~cm}^{-1} ;{ }^{1} \mathrm{H} \mathrm{NMR}\left(\mathrm{CDCl}_{3}, \mathrm{TMS}, 300 \mathrm{MHz}\right) \delta 1.26\left(3 \mathrm{H}, \mathrm{t}, J=7.2 \mathrm{~Hz}, \mathrm{CH}_{3}\right), 2.41(3 \mathrm{H}, \mathrm{s}$, Me), $2.46(3 \mathrm{H}, \mathrm{s}, \mathrm{Me}), 4.02(1 \mathrm{H}, \mathrm{dq}, J=10.8,7.2 \mathrm{~Hz}), 4.15(1 \mathrm{H}, \mathrm{dq}, J=10.8,7.2 \mathrm{~Hz}), 5.07$ $(1 \mathrm{H}, \mathrm{d}, J=6.3 \mathrm{~Hz}, \mathrm{NH}), 5.49(1 \mathrm{H}, \mathrm{d}, J=6.3 \mathrm{~Hz}, \mathrm{CH}), 7.06(1 \mathrm{H}, \mathrm{d}, J=2.4 \mathrm{~Hz}, \mathrm{Ar}), 7.21(2 \mathrm{H}, \mathrm{d}$, $J=8.4 \mathrm{~Hz}, \mathrm{Ar}), 7.27(1 \mathrm{H}, \mathrm{d}, J=2.4 \mathrm{~Hz}, \mathrm{Ar}), 7.52(2 \mathrm{H}, \mathrm{d}, J=8.4 \mathrm{~Hz}, \mathrm{Ar}) .{ }^{13} \mathrm{C} \mathrm{NMR}\left(\mathrm{CDCl}_{3}\right.$, TMS, 75.44 MHz) $\delta 14.1,19.5,21.4,47.5,60.9,103.3,122.2,122.3,126.6,127.7,129.1$, 129.28, 129.34, 138.7, 143.2, 145.4, 162.9, 165.7. MS (EI) m/e $300\left(\mathrm{M}^{+}-155,63.3\right), 285$ $\left(\mathrm{M}^{+}-170,100\right)$. Anal. Calcd. for $\mathrm{C}_{20} \mathrm{H}_{19} \mathrm{Cl}_{2} \mathrm{NO}_{5} \mathrm{~S}$ requires $\mathrm{C}, 52.64, \mathrm{H}, 4.20 ; \mathrm{N}, 3.07 \%$. Found: C, 52.92; H, 4.08; N, 2.94\%.

6-Bromo-2-methyl-4-(toluene-4-sulfonylamino)-4H-chromene-3-carboxylic acid ethyl ester (4f): a colorless solid: $\mathrm{mp} .150-151{ }^{\circ} \mathrm{C}$; IR $\left(\mathrm{CH}_{2} \mathrm{Cl}_{2}\right)$ v 3273, 1712, 1336, 1225, 1157 , $1071 \mathrm{~cm}^{-1} ;{ }^{1} \mathrm{H} \mathrm{NMR}\left(\mathrm{CDCl}_{3}, \mathrm{TMS}, 300 \mathrm{MHz}\right) \delta 1.24\left(3 \mathrm{H}, \mathrm{t}, J=7.2 \mathrm{~Hz}, \mathrm{CH}_{3}\right), 2.38(3 \mathrm{H}, \mathrm{s}$, Me), $2.40(3 \mathrm{H}, \mathrm{s}, \mathrm{Me}), 3.97(1 \mathrm{H}, \mathrm{dq}, J=10.8,7.2 \mathrm{~Hz}), 4.11(1 \mathrm{H}, \mathrm{dq}, J=10.8,7.2 \mathrm{~Hz}), 5.44$ $(1 \mathrm{H}, \mathrm{d}, J=6.6 \mathrm{~Hz}, \mathrm{NH}), 5.61(1 \mathrm{H}, \mathrm{d}, J=6.6 \mathrm{~Hz}, \mathrm{CH}), 6.88(1 \mathrm{H}, \mathrm{d}, J=9.0 \mathrm{~Hz}, \mathrm{Ar}), 7.17-7.19$ $(3 \mathrm{H}, \mathrm{m}, \mathrm{Ar}), 7.25-7.28(1 \mathrm{H}, \mathrm{m}, \mathrm{Ar}), 7.48(2 \mathrm{H}, \mathrm{d}, J=7.8 \mathrm{~Hz}, \mathrm{Ar}) .{ }^{13} \mathrm{C} \mathrm{NMR}\left(\mathrm{CDCl}_{3}, \mathrm{TMS}\right.$, 
75.44 MHz) $\delta 14.1,19.7,21.5,47.3,60.7,102.9,116.7,118.0,121.3,126.5,129.2,131.9$, 132.0, 138.9, 143.0, 149.7, 163.0, 166.1. MS (EI) m/e $465\left(\mathrm{M}^{+}, 1.3\right), 295\left(\mathrm{M}^{+}-170,100\right)$. Anal. Calcd. for $\mathrm{C}_{20} \mathrm{H}_{20} \mathrm{BrNO}_{5} \mathrm{~S}$ requires $\mathrm{C}, 51.51, \mathrm{H}, 4.32 ; \mathrm{N}, 3.00 \%$. Found: C, 51.39; H, 4.28; N, $2.85 \%$.

2-Methyl-6-nitro-4-(toluene-4-sulfonylamino)-4H-chromene-3-carboxylic acid ethyl ester (4g): a white solid: mp. $194-196{ }^{\circ} \mathrm{C}$; IR $\left(\mathrm{CH}_{2} \mathrm{Cl}_{2}\right) \vee 3312,1678,1339,1249,1158,1028$ $\mathrm{cm}^{-1} ;{ }^{1} \mathrm{H} \mathrm{NMR}\left(\mathrm{CDCl}_{3}, \mathrm{TMS}, 300 \mathrm{MHz}\right) \delta 1.30\left(3 \mathrm{H}, \mathrm{t}, J=7.2 \mathrm{~Hz}, \mathrm{CH}_{3}\right), 2.35(3 \mathrm{H}, \mathrm{s}, \mathrm{Me})$, $2.46(3 \mathrm{H}, \mathrm{s}, \mathrm{Me}), 4.06-4.25\left(2 \mathrm{H}, \mathrm{m}, \mathrm{CH}_{2}\right), 5.42(1 \mathrm{H}, \mathrm{d}, J=6.0 \mathrm{~Hz}, \mathrm{NH}), 5.52(1 \mathrm{H}, \mathrm{d}, J=6.6$ $\mathrm{Hz}, \mathrm{CH}), 7.12-7.16(3 \mathrm{H}, \mathrm{m}, \mathrm{Ar}), 7.41-7.45(2 \mathrm{H}, \mathrm{m}, \mathrm{Ar}), 7.97(1 \mathrm{H}, \mathrm{d}, J=2.7 \mathrm{~Hz}, \mathrm{Ar}), 8.07$ (1H, $\mathrm{dd}, J=9.0,2.7 \mathrm{~Hz}, \mathrm{Ar}) .{ }^{13} \mathrm{C} \mathrm{NMR}\left(\mathrm{CDCl}_{3}\right.$, TMS, 75.44 MHz) $\delta 14.1,19.6,21.3,47.4,61.1$, $103.4,117.3,119.9,124.6,126.1,126.5,129.3,138.9,143.3,143.8,154.9,162.8,165.7 . \mathrm{MS}$ (EI) m/e $432\left(\mathrm{M}^{+}, 0.51\right), 262\left(\mathrm{M}^{+}-170,100\right)$. Anal. Calcd. for $\mathrm{C}_{20} \mathrm{H}_{20} \mathrm{~N}_{2} \mathrm{O}_{7} \mathrm{~S}$ requires $\mathrm{C}, 55.55$, H, 4.66; N, 6.48\%. Found: C, 55.48; H, 4.60; N, 6.25\%.

\section{Typical Reaction Procedure for DABCO-catalyzed Reaction of Penta-3,4-dien-2-one 2b} with Salicyl $N$-Tosylimine 1a.

To a flame-dried Schlenk tube at room temperature was in turn added molecular sieve 4A (100 mg), DABCO (5.6 mg, $0.05 \mathrm{mmol})$, salicyl $N$-tosylimine 1a $(138 \mathrm{mg}, 0.50 \mathrm{mmol})$, THF (2.0 $\mathrm{mL})$ and penta-3,4-dien-2-one $2 \mathbf{b}(55 \mu \mathrm{L}, 0.60 \mathrm{mmol})$ and the reaction mixture was further stirred at room temperature. The reaction was monitored by TLC plate. When 1a disappeared, the solvent was removed under reduced pressure and the residue was purified by a flash 
chromatography (eluent: EtOAc:Petroleum ether $=1: 4-2: 1)$ to yield 5a $(156 \mathrm{mg}, 87 \%)$ as a colorless solid.

$N$-(3-Acetyl-2-methyl-4H-chromen-4-yl)-4-methyl-benzenesulfonamide (5a): a colorless solid: mp. $146-147{ }^{\circ} \mathrm{C}$; IR $\left(\mathrm{CH}_{2} \mathrm{Cl}_{2}\right)$ v 3263, 1680, 1573, 1381, 1244, 1222, $1156 \mathrm{~cm}^{-1} ;{ }^{1} \mathrm{H}$ NMR $\left(\mathrm{CDCl}_{3}, \mathrm{TMS}, 300 \mathrm{MHz}\right) \delta 2.28(3 \mathrm{H}, \mathrm{s}, \mathrm{Me}), 2.36(3 \mathrm{H}, \mathrm{s}, \mathrm{Me}), 2.40(3 \mathrm{H}, \mathrm{s}, \mathrm{Me}), 4.99$ $(1 \mathrm{H}, \mathrm{d}, J=7.2 \mathrm{~Hz}, \mathrm{NH}), 5.59(1 \mathrm{H}, \mathrm{d}, J=7.2 \mathrm{~Hz}, \mathrm{CH}), 6.93-6.98(1 \mathrm{H}, \mathrm{m}, \mathrm{Ar}), 7.02-7.05(1 \mathrm{H}, \mathrm{m}$, Ar), 7.17-7.25 (4H, m, Ar), $7.53(2 \mathrm{H}, \mathrm{d}, J=8.4 \mathrm{~Hz}, \mathrm{Ar}) .{ }^{13} \mathrm{C} \mathrm{NMR}\left(\mathrm{CDCl}_{3}, \mathrm{TMS}, 75.44 \mathrm{MHz}\right)$ $\delta 20.3,21.4,29.7,48.1,111.8,116.2,120.2,124.5(2 \mathrm{C}), 126.7,129.0,129.3,138.7,143.0$, 150.3, 162.2, 198.2. MS (EI) m/e $357\left(\mathrm{M}^{+}\right.$, 4.32), $187\left(\mathrm{M}^{+}-170,100\right)$. Anal. Calcd. for $\mathrm{C}_{19} \mathrm{H}_{19} \mathrm{NO}_{4} \mathrm{~S}$ requires C, 63.85, H, 5.36; N, 3.92\%. Found: C, 64.12; H, 5.53; N, 3.84\%.

$N$-(3-Acetyl-8-methoxy-2-methyl-4H-chromen-4-yl)-4-methyl-benzenesulfonamide (5b): a white solid: mp. $163-164{ }^{\circ} \mathrm{C}$; IR $\left(\mathrm{CH}_{2} \mathrm{Cl}_{2}\right)$ v 3263, 1678, 1565, 1491, 1243, 1211, 1155, 1095 $\mathrm{cm}^{-1} ;{ }^{1} \mathrm{H}$ NMR $\left(\mathrm{CDCl}_{3}, \mathrm{TMS}, 300 \mathrm{MHz}\right) \delta 2.28(3 \mathrm{H}, \mathrm{s}, \mathrm{Me}), 2.30(3 \mathrm{H}, \mathrm{s}, \mathrm{Me}), 2.40(3 \mathrm{H}, \mathrm{s}, \mathrm{Me})$, $3.87(3 \mathrm{H}, \mathrm{s}, \mathrm{MeO}), 4.98(1 \mathrm{H}, \mathrm{d}, J=7.5 \mathrm{~Hz}, \mathrm{NH}), 5.64(1 \mathrm{H}, \mathrm{d}, J=7.5 \mathrm{~Hz}, \mathrm{CH}), 6.79-6.96(3 \mathrm{H}$, $\mathrm{m}, \mathrm{Ar}), 7.20(2 \mathrm{H}, \mathrm{d}, J=8.4 \mathrm{~Hz}, \mathrm{Ar}), 7.59(2 \mathrm{H}, \mathrm{d}, J=8.4 \mathrm{~Hz}, \mathrm{Ar}) .{ }^{13} \mathrm{C} \mathrm{NMR}\left(\mathrm{CDCl}_{3}, \mathrm{TMS}\right.$, 75.44 MHz) $\delta 19.7,21.4,29.5,48.0,55.8,110.8,111.3,120.5,121.6,124.3,126.7,129.3$, 138.9, 139.8, 143.0, 147.1, 161.6, 198.4. MS (EI) m/e $387\left(\mathrm{M}^{+}, 2.54\right), 217\left(\mathrm{M}^{+}-170,100\right)$. Anal. Calcd. for $\mathrm{C}_{20} \mathrm{H}_{21} \mathrm{NO}_{5} \mathrm{~S}$ requires C, 62.00, H, 5.46; N, 3.62\%. Found: C, 62.05; H, 5.51; $\mathrm{N}, 3.44 \%$. 
$N$-(3-Acetyl-7-methoxy-2-methyl-4H-chromen-4-yl)-4-methyl-benzenesulfonamide (5c): a colorless solid: $\mathrm{mp} .150-152{ }^{\circ} \mathrm{C}$; IR $\left(\mathrm{CH}_{2} \mathrm{Cl}_{2}\right) \vee 3259,1665,1610,1330,1280,1212,1162$ $\mathrm{cm}^{-1} ;{ }^{1} \mathrm{H} \mathrm{NMR}\left(\mathrm{CDCl}_{3}, \mathrm{TMS}, 300 \mathrm{MHz}\right) \delta 2.28(3 \mathrm{H}, \mathrm{s}, \mathrm{Me}), 2.35(3 \mathrm{H}, \mathrm{s}, \mathrm{Me}), 2.40(3 \mathrm{H}, \mathrm{s}, \mathrm{Me})$, $3.78(3 \mathrm{H}, \mathrm{s}, \mathrm{MeO}), 4.83(1 \mathrm{H}, \mathrm{d}, J=7.5 \mathrm{~Hz}, \mathrm{NH}), 5.55(1 \mathrm{H}, \mathrm{d}, J=7.5 \mathrm{~Hz}, \mathrm{CH}), 6.51-6.55(2 \mathrm{H}$, m, Ar), $7.13(1 \mathrm{H}, \mathrm{d}, J=8.4 \mathrm{~Hz}, \mathrm{Ar}), 7.20(2 \mathrm{H}, \mathrm{d}, J=8.1 \mathrm{~Hz}, \mathrm{Ar}), 7.55(2 \mathrm{H}, \mathrm{d}, J=8.1 \mathrm{~Hz}, \mathrm{Ar})$. ${ }^{13} \mathrm{C} \mathrm{NMR}\left(\mathrm{CDCl}_{3}\right.$, TMS, 75.44 MHz) $\delta 20.3,21.4,29.7,48.0,55.4,100.6,111.6,112.2,112.5$, 126.8, 129.3, 130.0, 138.8, 143.0, 151.2, 160.1, 161.7, 198.4. MS (EI) m/e 387 (M+ $\mathrm{M}^{+}$. .00$), 91$ $\left(\mathrm{M}^{+}-296,100\right)$. Anal. Calcd. for $\mathrm{C}_{20} \mathrm{H}_{21} \mathrm{NO}_{5} \mathrm{~S}$ requires $\mathrm{C}, 62.00, \mathrm{H}, 5.46 ; \mathrm{N}, 3.62 \%$. Found: $\mathrm{C}$, $62.20 ; \mathrm{H}, 5.56 ; \mathrm{N}, 3.44 \%$.

$N$-(3-Acetyl-6-methoxy-2-methyl-4H-chromen-4-yl)-4-methyl-benzenesulfonamide (5d): a colorless solid: mp. $134-135{ }^{\circ} \mathrm{C}$; IR $\left(\mathrm{CH}_{2} \mathrm{Cl}_{2}\right) \vee 3263,1679,1576,1498,1338,1220,1156$ $\mathrm{cm}^{-1} ;{ }^{1} \mathrm{H} \mathrm{NMR}\left(\mathrm{CDCl}_{3}, \mathrm{TMS}, 300 \mathrm{MHz}\right) \delta 2.30(3 \mathrm{H}, \mathrm{s}, \mathrm{Me}), 2.36(3 \mathrm{H}, \mathrm{s}, \mathrm{Me}), 2.39(3 \mathrm{H}, \mathrm{s}, \mathrm{Me})$, $3.62(3 \mathrm{H}, \mathrm{s}, \mathrm{MeO}), 5.04(1 \mathrm{H}, \mathrm{d}, J=6.6 \mathrm{~Hz}, \mathrm{NH}), 5.55(1 \mathrm{H}, \mathrm{d}, J=6.6 \mathrm{~Hz}, \mathrm{CH}), 6.65(1 \mathrm{H}, \mathrm{d}, J=$ 2.7 Hz, Ar), $6.77(1 \mathrm{H}, \mathrm{dd}, J=8.7,2.7 \mathrm{~Hz}, \mathrm{Ar}), 6.97(1 \mathrm{H}, \mathrm{d}, J=8.7 \mathrm{~Hz}, \mathrm{Ar}), 7.18(2 \mathrm{H}, \mathrm{d}, J=8.4$ $\mathrm{Hz}, \mathrm{Ar}), 7.52(2 \mathrm{H}, \mathrm{d}, J=8.4 \mathrm{~Hz}, \mathrm{Ar}) .{ }^{13} \mathrm{C} \mathrm{NMR}\left(\mathrm{CDCl}_{3}\right.$, TMS, $\left.75.44 \mathrm{MHz}\right) \delta 20.5,21.4,29.8$, $48.6,55.3,111.1,111.9,116.3,117.3,120.4,126.6,129.2,138.9,142.9,144.5,156.0,162.5$, 198.2. MS (EI) m/e $387\left(\mathrm{M}^{+}, 6.98\right), 217\left(\mathrm{M}^{+}-170,100\right)$. Anal. Calcd. for $\mathrm{C}_{20} \mathrm{H}_{21} \mathrm{NO}_{5} \mathrm{~S}$ requires C, 62.00, H, 5.46; N, 3.62\%. Found: C, 62.05; H, 5.44; N, 3.39\%. 
$\mathrm{cm}^{-1} ;{ }^{1} \mathrm{H}$ NMR $\left(\mathrm{CDCl}_{3}, \mathrm{TMS}, 300 \mathrm{MHz}\right) \delta 2.07$ (3H, s, Me), $2.34(3 \mathrm{H}, \mathrm{s}, \mathrm{Me}), 2.35(3 \mathrm{H}, \mathrm{s}, \mathrm{Me})$, $2.39(3 \mathrm{H}, \mathrm{s}, \mathrm{Me}), 5.22(1 \mathrm{H}, \mathrm{d}, J=7.2 \mathrm{~Hz}, \mathrm{NH}), 5.50(1 \mathrm{H}, \mathrm{d}, J=7.2 \mathrm{~Hz}, \mathrm{CH}), 6.76(1 \mathrm{H}, \mathrm{s}, \mathrm{Ar})$, 6.88-6.98 (2H, m, Ar), $7.16(2 \mathrm{H}, \mathrm{d}, J=8.4 \mathrm{~Hz}, \mathrm{Ar}), 7.50(2 \mathrm{H}, \mathrm{d}, J=8.4 \mathrm{~Hz}, \mathrm{Ar}) .{ }^{13} \mathrm{C} \mathrm{NMR}$ $\left(\mathrm{CDCl}_{3}, \mathrm{TMS}, 75.44 \mathrm{MHz}\right) \delta 20.4(2 \mathrm{C}), 21.4,29.8,48.4,111.8,115.9,119.4,126.7,129.2$, 129.3, 129.7, 133.9, 138.9, 142.8, 148.4, 162.4, 198.3. MS (EI) m/e $371\left(\mathrm{M}^{+}, 1.63\right), 201$ $\left(\mathrm{M}^{+}-170,100\right)$. Anal. Calcd. for $\mathrm{C}_{20} \mathrm{H}_{21} \mathrm{NO}_{4} \mathrm{~S}$ requires $\mathrm{C}, 64.67, \mathrm{H}, 5.70 ; \mathrm{N}, 3.77 \%$. Found: $\mathrm{C}$, $64.95 ; \mathrm{H}, 5.56 ; \mathrm{N}, 3.74 \%$.

$N$-(3-Acetyl-6-bromo-2-methyl-4H-chromen-4-yl)-4-methyl-benzenesulfonamide (5f): a colorless solid: mp. $150-152{ }^{\circ} \mathrm{C}$; IR $\left(\mathrm{CH}_{2} \mathrm{Cl}_{2}\right)$ v 3259, 1682, 1571, 1480, 1334, 1243, 1225 , $1157 \mathrm{~cm}^{-1} ;{ }^{1} \mathrm{H}$ NMR $\left(\mathrm{CDCl}_{3}, \mathrm{TMS}, 300 \mathrm{MHz}\right) \delta 2.37$ (3H, s, Me), 2.39 (3H, s, Me), $2.42(3 \mathrm{H}$, s, Me), $5.06(1 \mathrm{H}, \mathrm{d}, J=6.9 \mathrm{~Hz}, \mathrm{NH}), 5.46(1 \mathrm{H}, \mathrm{d}, J=6.9 \mathrm{~Hz}, \mathrm{CH}), 6.91(1 \mathrm{H}, \mathrm{d}, J=8.7 \mathrm{~Hz}, \mathrm{Ar})$, $7.06(1 \mathrm{H}, \mathrm{d}, J=2.4 \mathrm{~Hz}, \mathrm{Ar}), 7.20(2 \mathrm{H}, \mathrm{d}, J=8.4 \mathrm{~Hz}, \mathrm{Ar}), 7.27(1 \mathrm{H}, \mathrm{dd}, J=8.7,2.4 \mathrm{~Hz}, \mathrm{Ar})$, $7.49(2 \mathrm{H}, \mathrm{d}, J=8.4 \mathrm{~Hz}, \mathrm{Ar}) .{ }^{13} \mathrm{C} \mathrm{NMR}\left(\mathrm{CDCl}_{3}, \mathrm{TMS}, 75.44 \mathrm{MHz}\right) \delta 20.2,21.5,29.7,47.6$, $111.5,116.4,118.0,121.6,126.4,129.4,131.6,131.8,138.3,143.2,149.3,162.2,198.0 . \mathrm{MS}$ (EI) m/e $435\left(\mathrm{M}^{+}, 0.52\right), 265\left(\mathrm{M}^{+}-170,100\right)$. Anal. Calcd. for $\mathrm{C}_{19} \mathrm{H}_{18} \mathrm{BrNO}_{4} \mathrm{~S}$ requires $\mathrm{C}, 52.30$, H, 4.16; N, 3.21\%. Found: C, 52.25; H, 4.07; N, $2.98 \%$.

$N$-(3-Acetyl-6,8-dichloro-2-methyl-4H-chromen-4-yl)-4-methyl-benzenesulfonamide (5g): a colorless solid: mp. $155-157{ }^{\circ} \mathrm{C}$; IR $\left(\mathrm{CH}_{2} \mathrm{Cl}_{2}\right) \vee 3258,1686,1573,1464,1251,1206,1157$ $\mathrm{cm}^{-1} ;{ }^{1} \mathrm{H} \mathrm{NMR}\left(\mathrm{CDCl}_{3}, \mathrm{TMS}, 300 \mathrm{MHz}\right) \delta 2.34(3 \mathrm{H}, \mathrm{s}, \mathrm{Me}), 2.41$ (3H, s, Me), $2.43(3 \mathrm{H}, \mathrm{s}, \mathrm{Me})$, $5.17(1 \mathrm{H}, \mathrm{d}, J=7.2 \mathrm{~Hz}, \mathrm{NH}), 5.47(1 \mathrm{H}, \mathrm{d}, J=7.2 \mathrm{~Hz}, \mathrm{CH}), 6.91(1 \mathrm{H}, \mathrm{d}, J=2.4 \mathrm{~Hz}, \mathrm{Ar})$, 
7.20-7.24 (3H, m, Ar), 7.50-7.53 (2H, m, Ar). ${ }^{13} \mathrm{C} \mathrm{NMR}\left(\mathrm{CDCl}_{3}, \mathrm{TMS}, 75.44 \mathrm{MHz}\right) \delta 20.2$, $21.5,29.9,47.7,112.0,122.3,122.5,126.6,127.3,128.9,129.3,129.5,138.2,143.5,145.2$, 162.0, 197.6. MS (EI) $\mathrm{m} / \mathrm{e} 425\left(\mathrm{M}^{+}, 0.85\right), 255\left(\mathrm{M}^{+}-170,100\right)$. Anal. Calcd. for $\mathrm{C}_{19} \mathrm{H}_{17} \mathrm{Cl}_{2} \mathrm{NO}_{4} \mathrm{~S}$ requires C, 53.53; H, 4.02; N, 3.29\%. Found: C, 53.43; H, 3.98; N, 3.09\%.

$N$-(3-Acetyl-2-methyl-6-nitro-4H-chromen-4-yl)-4-methyl-benzenesulfonamide (5h): a white solid: mp. $157-159{ }^{\circ} \mathrm{C}$; IR $\left(\mathrm{CH}_{2} \mathrm{Cl}_{2}\right) \vee 3263,1686,1527,1341,1245,1213,1157 \mathrm{~cm}^{-1}$; ${ }^{1} \mathrm{H}$ NMR $\left(\mathrm{CDCl}_{3}\right.$, TMS, $\left.300 \mathrm{MHz}\right) \delta 2.34$ (3H, s, Me), 2.36 (3H, s, Me), 2.39 (3H, s, Me), 5.53 $(1 \mathrm{H}, \mathrm{d}, J=7.2 \mathrm{~Hz}, \mathrm{NH}), 5.84(1 \mathrm{H}, \mathrm{d}, J=7.2 \mathrm{~Hz}, \mathrm{CH}), 7.10-7.13(3 \mathrm{H}, \mathrm{m}, \mathrm{Ar}), 7.44(2 \mathrm{H}, \mathrm{d}, J=$ $8.4 \mathrm{~Hz}, \mathrm{Ar}), 7.84(1 \mathrm{H}, \mathrm{d}, J=3.0 \mathrm{~Hz}, \mathrm{Ar}), 7.99-8.03(1 \mathrm{H}, \mathrm{m}, \mathrm{Ar}) .{ }^{13} \mathrm{C} \mathrm{NMR}\left(\mathrm{CDCl}_{3}, \mathrm{TMS}\right.$, 75.44 MHz) $\delta 20.1,21.3,30.0,47.4,112.1,117.3,120.3,124.4,125.6,126.4,129.4,138.4$, 143.5, 143.6, 154.5, 161.7, 197.6. MS (EI) m/e 402 ( $\left.\mathrm{M}^{+}, 1.46\right), 232$ ( $\left.\mathrm{M}^{+}-170,100\right)$. Anal. Calcd. for $\mathrm{C}_{19} \mathrm{H}_{18} \mathrm{~N}_{2} \mathrm{O}_{6} \mathrm{~S}$ requires $\mathrm{C}, 56.71 ; \mathrm{H}, 4.51 ; \mathrm{N}, 6.96 \%$. Found: C, 57.00; H, 4.73; N, $6.86 \%$.

$N$-(2-Acetyl-3-methyl-1H-benzo[f]chromen-1-yl)-4-methyl-benzenesulfonamide (5i): a yellow solid: mp. $160-161{ }^{\circ} \mathrm{C}$; IR $\left(\mathrm{CH}_{2} \mathrm{Cl}_{2}\right) \vee 3270,2924,1682,1642,1594,1332,1231,1155$ $\mathrm{cm}^{-1} ;{ }^{1} \mathrm{H}$ NMR $\left(\mathrm{CDCl}_{3}, \mathrm{TMS}, 300 \mathrm{MHz}\right) \delta 2.16(3 \mathrm{H}, \mathrm{s}, \mathrm{Me}), 2.44(3 \mathrm{H}, \mathrm{s}, \mathrm{Me}), 2.48(3 \mathrm{H}, \mathrm{s}, \mathrm{Me})$, $5.67(1 \mathrm{H}, \mathrm{d}, J=4.8 \mathrm{~Hz}, \mathrm{NH}), 6.05(1 \mathrm{H}, \mathrm{d}, J=4.8 \mathrm{~Hz}, \mathrm{CH}), 6.70(2 \mathrm{H}, \mathrm{d}, J=8.4 \mathrm{~Hz}, \mathrm{Ar}), 7.07$ $(2 \mathrm{H}, \mathrm{d}, J=8.4 \mathrm{~Hz}, \mathrm{Ar}), 7.19(1 \mathrm{H}, \mathrm{d}, J=9.0 \mathrm{~Hz}, \mathrm{Ar}), 7.29-7.38(2 \mathrm{H}, \mathrm{m}, \mathrm{Ar}), 7.59-7.67(2 \mathrm{H}, \mathrm{m}$, Ar), $7.89(1 \mathrm{H}, \mathrm{d}, J=8.1 \mathrm{~Hz}, \mathrm{Ar}) .{ }^{13} \mathrm{C} \mathrm{NMR}\left(\mathrm{CDCl}_{3}, \mathrm{TMS}, 75.44 \mathrm{MHz}\right) \delta 20.9,21.2,31.1,45.3$, $110.8,113.5,116.4,122.7,124.8,126.2,127.1,128.0,128.3,130.2,130.66,130.69,137.7$, 
141.9, 149.4, 162.6, 197.9. MS (EI) m/e $407\left(\mathrm{M}^{+}, 2.91\right), 91\left(\mathrm{M}^{+}-316,100\right)$. Anal. Calcd. for $\mathrm{C}_{23} \mathrm{H}_{21} \mathrm{NO}_{4} \mathrm{~S}$ requires C, 67.79; H, 5.19; N, 3.44\%. Found: C, 67.73; H, 5.16; N, 3.26\%.

Typical Reaction Procedure for DABCO-catalyzed Reaction of Penta-3,4-dien-2-one 2b with Salicylaldehyde.

To a solution of salicylaldehyde $(55 \mu \mathrm{L}, 0.50 \mathrm{mmol})$ and penta-3,4-dien-2-one $2 \mathbf{b}$ ( $55 \mu \mathrm{L}, 0.60$ $\mathrm{mmol})$ in THF $(2.0 \mathrm{~mL})$ at room temperature was added DABCO $(18 \mathrm{mg}, 0.0625 \mathrm{mmol})$ and the reaction mixture was further stirred at room temperature. The reaction was monitored by TLC plate. When salicylaldehyde disappeared, the solvent was removed under reduced pressure and the residue was purified by a flash chromatography (eluent: EtOAc:Petroleum ether $=1: 10)$ to yield $\mathbf{1 3 a}(78 \mathrm{mg}, 76 \%)$ as a colorless liquid.

E- 2-(1-Methyl-3-oxo-but-1-enyloxy)-benzaldehyde (13a): a colorless liquid: $\mathrm{IR}\left(\mathrm{CH}_{2} \mathrm{Cl}_{2}\right) \mathrm{v}$ 2855, 1693, 1596, 1578, 1389, 1274, 1238, 1209, $1151 \mathrm{~cm}^{-1} ;{ }^{1} \mathrm{H}$ NMR $\left(\mathrm{CDCl}_{3}, \mathrm{TMS}, 300\right.$ MHz) $\delta 2.04(3 \mathrm{H}, \mathrm{s}, \mathrm{Me}), 2.54(3 \mathrm{H}, \mathrm{s}, \mathrm{Me}), 5.25(1 \mathrm{H}, \mathrm{s}), 7.10 \square 7.13(1 \mathrm{H}, \mathrm{m}, \mathrm{Ar}), 7.37-7.42$ (1H, m, Ar), 7.65-7.70 (1H, m, Ar), 7.95-7.98 (1H, m, Ar), 10.14 (1H, d, J=0.6 Hz, CHO).

${ }^{13} \mathrm{C}$ NMR $\left(\mathrm{CDCl}_{3}\right.$, TMS, 75.44 MHz) $\delta 18.6,32.0,105.3,122.8,126.3,128.1,129.1,135.9$, 155.3, 172.0, 188.1, 196.9. MS (EI) m/e $205\left(\mathrm{M}^{+}+1,64.7\right), 204\left(\mathrm{M}^{+}, 17.7\right), 161\left(\mathrm{M}^{+}-43,100\right)$. HRMS Calcd. for $\mathrm{C}_{12} \mathrm{H}_{12} \mathrm{O}_{3} \mathrm{Na}^{+}$requires 227.0679, Found 227.0690. 

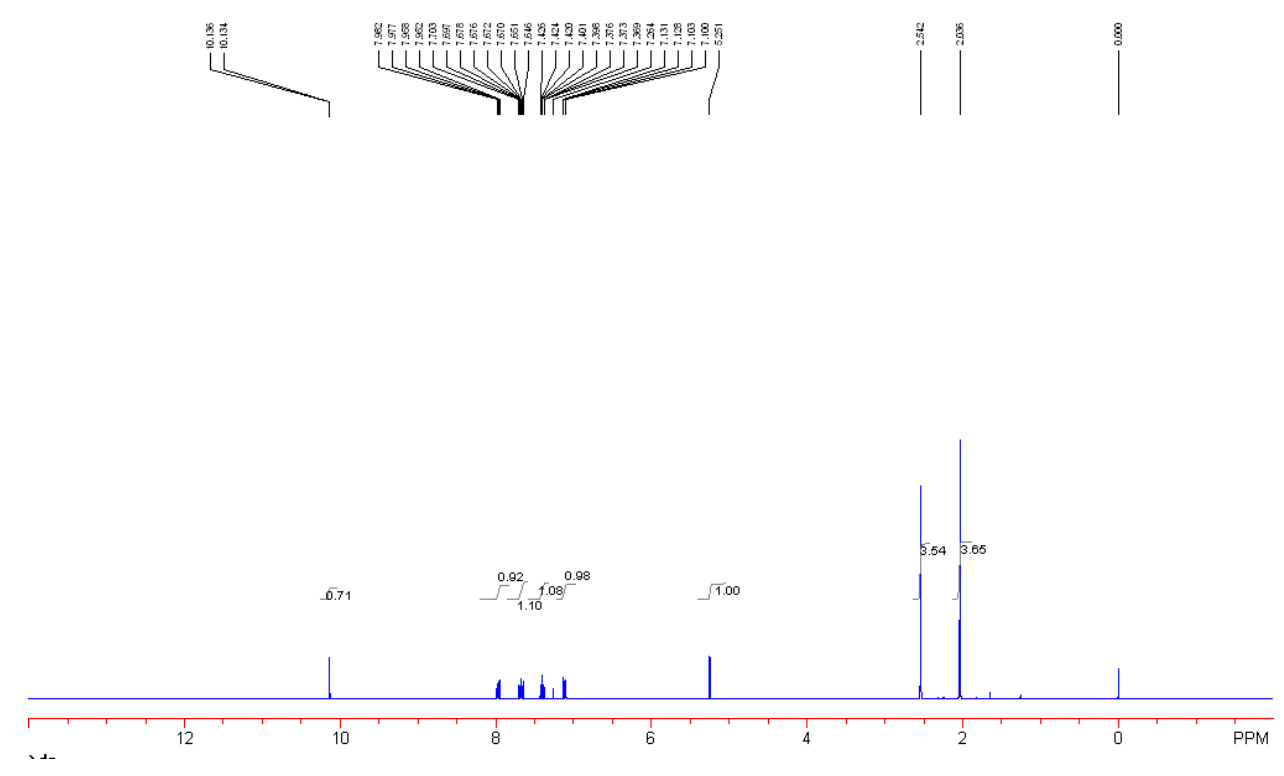

Typical Reaction Procedure for DABCO-catalyzed Reaction of Ethyl 2,3-butadienoate 2a with Salicylaldehyde.

To a solution of salicylaldehyde $(55 \mu \mathrm{L}, 0.50 \mathrm{mmol})$ and ethyl 2,3-butadienoate $2 \mathbf{a}(69 \mu \mathrm{L}$, $0.60 \mathrm{mmol})$ in THF $(2.0 \mathrm{~mL})$ at room temperature was added DABCO $(18 \mathrm{mg}, 0.0625 \mathrm{mmol})$ and the reaction mixture was further stirred at room temperature. The reaction was monitored by TLC plate. When salicylaldehyde disappeared, the solvent was removed under reduced pressure and the residue was purified by a flash chromatography (eluent: EtOAc:Petroleum ether $=1: 10)$ to yield $\mathbf{1 3 b}(83 \mathrm{mg}, 71 \%)$ as a colorless liquid.

E- 3-(2-Formyl-phenoxy)-but-2-enoic acid ethyl ester (13b): a colorless liquid: $\mathrm{IR}\left(\mathrm{CHCl}_{3}\right)$ v 2981, 1712, 1639, 1601, 1387, 1274, 1247, 1212, $1131 \mathrm{~cm}^{-1} ;{ }^{1} \mathrm{H}$ NMR $\left(\mathrm{CDCl}_{3}, \mathrm{TMS}, 300\right.$ MHz) $\delta 1.21\left(3 \mathrm{H}, \mathrm{t}, J=7.2 \mathrm{~Hz}, \mathrm{CH}_{3}\right), 2.57(3 \mathrm{H}, \mathrm{s}, \mathrm{Me}), 4.10\left(2 \mathrm{H}, \mathrm{q}, J=7.2 \mathrm{~Hz}, \mathrm{CH}_{2}\right), 4.84$ $(1 \mathrm{H}, \mathrm{s}), 7.11-7.14(1 \mathrm{H}, \mathrm{m}, \mathrm{Ar}), 7.35-7.40(1 \mathrm{H}, \mathrm{m}, \mathrm{Ar}), 7.63-7.69(1 \mathrm{H}, \mathrm{m}, \mathrm{Ar})$, 7.94-7.97 (1H, m, Ar), $10.14(1 \mathrm{H}, \mathrm{d}, J=0.6 \mathrm{~Hz}, \mathrm{CHO}) .{ }^{13} \mathrm{C} \mathrm{NMR}\left(\mathrm{CDCl}_{3}, \mathrm{TMS}, 75.44 \mathrm{MHz}\right) \delta 14.2,18.0,59.8$, 
98.0, 122.8, 126.3, 128.1, 129.2, 135.9, 155.4, 166.9, 172.6, 188.2. MS (EI) m/e $234\left(\mathrm{M}^{+}\right.$, 7.23), $147\left(\mathrm{M}^{+}-87,100\right)$. HRMS Calcd. for $\mathrm{C}_{13} \mathrm{H}_{14} \mathrm{O}_{4} \mathrm{Na}^{+}$requires 257.0784, Found 257.0793.
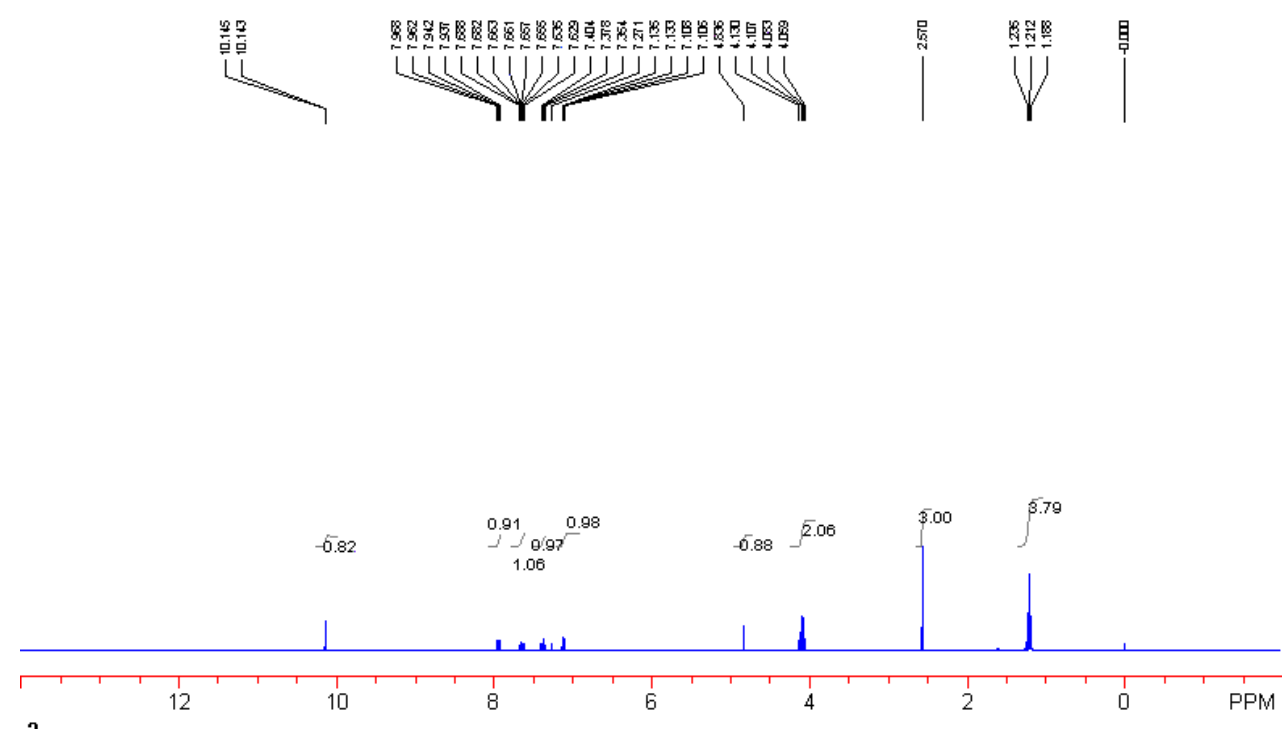

Reference:

(1) Wynne, J. H.; Price, S. E.; Rorer, J. R.; Stalick, W. M. Synth. Commun.. 2003, 33, 341.

(2) Anderson, J. C.; Cubbon, R. J.; Harling, J. D. Tetrahedron: Asymmetry 2001, 12, 923.

(3) Buono, G. Synthesis 1981, 272. 


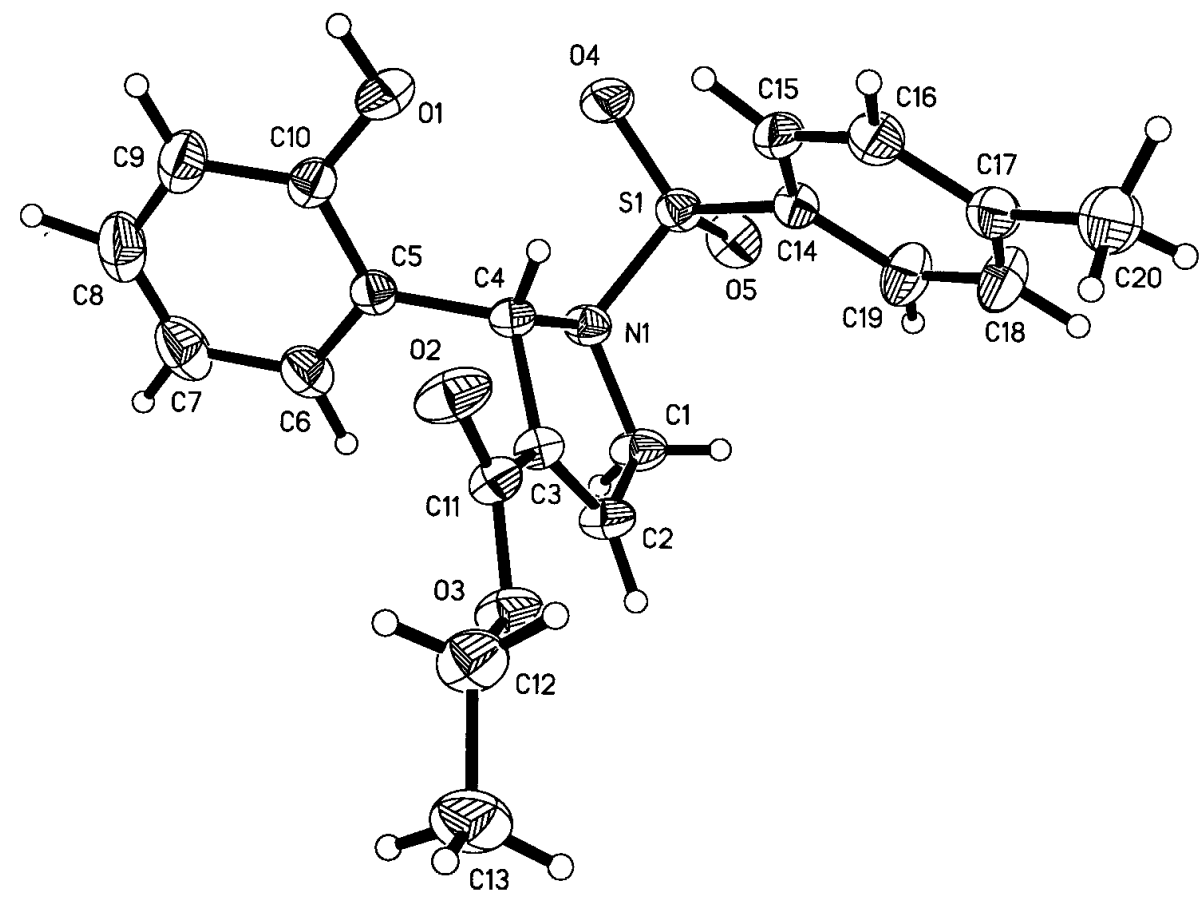

Figure 1. The ORTEP draw of 3a.

The crystal data of 3a has been deposited in CCDC with number 266106. Empirical Formula: $\mathrm{C}_{20} \mathrm{H}_{21} \mathrm{NO}_{5} \mathrm{~S}$; Formula Weight: 387.44; Temperature: 293(2)K; Crystal Dimensions: 0.516 x 0.427 x $0.344 \mathrm{~mm}$; Crystal System: Orthorhombic; Space group:

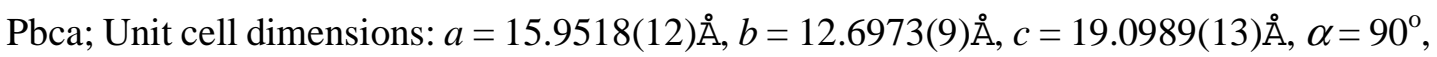
$\beta=90^{\circ}, \gamma=90^{\circ}, V=3868.4(5) \AA^{3} ; Z=8 ; D_{\text {calc. }}=1.330 \mathrm{mg} / \mathrm{m}^{3} ; F_{000}=1632 ;$ Final R induces $[\mathrm{I}>2 \sigma(\mathrm{I})]: R_{1}=0.0435, R_{2}=0.1235$. 
Table 1. Crystal data and structure refinement for cd24432.

Identification code

Empirical formula

Formula weight

Temperature

Wave length

Crystal system, space group

Unit cell dimensions

Volume

Z, Calculated density

Absorption coefficient

$\mathrm{F}(000)$

Crystal size

Theta range for data collection

Limiting indices

Reflections collected / unique

Completeness to theta $=27.00$

Absorption correction

Max. and min. transmission

lef inement method

Data / restraints / parameters $\operatorname{cd} 24432$

C20 H21 N05 S

387.44

$293(2) \mathrm{K}$

$0.71073 \mathrm{~A}$

Orthorhombic, Pbca

$\mathrm{a}=15.9518(12)$ A alpha $=90 \mathrm{deg}$.

$\mathrm{b}=12.6973(9) \mathrm{A}$ beta $=90 \mathrm{deg}$.

$\mathrm{c}=19.0989(13) \mathrm{A}$ gamma $=90$ deg.

$3868.4(5)$ A 3

8, $\quad 1.330 \mathrm{Mg} / \mathrm{m}^{-3}$

$0.198 \mathrm{~mm}^{-1}$

1632

$0.516 \times 0.427 \times 0.344 \mathrm{mInI}$

2.13 to $27.00 \mathrm{deg}$.

$-20<=\mathrm{h}<=14, \quad-15<=\mathrm{k}<=16, \quad-19<=1<=24$

$21440 / 4224[\mathrm{R}($ int $)=0.0476]$

$99.9 \%$

Timp i ri çakl

1.00000 and 0.62973

Ful-malrix lesst-squares on $\mathrm{F}^{\wedge} 2$

$4224 / 0 / 318$ 


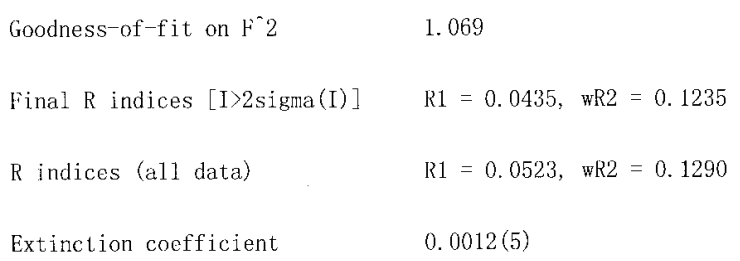


Table 2. Atomic coordinates ( $\mathrm{x} 10^{\wedge} 4$ ) and equivalent isotropic displacement parameters (A $2 \times 10^{\circ} 3$ ) for cd24432.

$\mathrm{U}(\mathrm{eq})$ is defined as one third of the trace of the orthogonalized Uij tensor.

\begin{tabular}{|c|c|c|c|c|}
\hline & $x$ & y & $z$ & $\mathrm{U}(\mathrm{eq})$ \\
\hline$S(1)$ & $2407(1)$ & $8769(1)$ & $1605(1)$ & $41(1)$ \\
\hline $0(1)$ & $770(1)$ & $10326(1)$ & $295(1)$ & $57(1)$ \\
\hline $0(2)$ & $82(1)$ & $7780(1)$ & $-236(1)$ & $65(1)$ \\
\hline $0(3)$ & $601(1)$ & $6206(1)$ & $-496(1)$ & $54(1)$ \\
\hline $0(4)$ & $2186(1)$ & $9852(1)$ & $1533(1)$ & $54(1)$ \\
\hline $0(5)$ & $3181(1)$ & $8481(1)$ & $1925(1)$ & $59(1)$ \\
\hline$N(1)$ & $2420(1)$ & $8273(1)$ & $817(1)$ & $40(1)$ \\
\hline$C(1)$ & $2697(1)$ & $7163(1)$ & $745(1)$ & $51(1)$ \\
\hline$c(2)$ & $2024(1)$ & $6672(1)$ & $323(1)$ & $46(1)$ \\
\hline$C(3)$ & $1449(1)$ & 7367 (1) & $136(1)$ & $39(1)$ \\
\hline$C(4)$ & $1655(1)$ & $8464(1)$ & $386(1)$ & $35(1)$ \\
\hline$C(5)$ & $1803(1)$ & $9242(1)$ & $-198(1)$ & $10(1)$ \\
\hline$c(6)$ & $2409(1)$ & $9079(2)$ & $-702(1)$ & $56(1)$ \\
\hline$C(7)$ & $2530(2)$ & $9804(2)$ & $-1240(1)$ & $74(1)$ \\
\hline$C(8)$ & $2033(2)$ & $10675(2)$ & $-1274(1)$ & $76(1)$ \\
\hline$C(9)$ & $1123(2)$ & $10857(2)$ & $-779(1)$ & $63(1)$ \\
\hline$C(10)$ & $1319(1)$ & $10152(1)$ & $-232(1)$ & $44(1)$ \\
\hline$C(11)$ & $646(1)$ & $7159(1)$ & $-218(1)$ & $43(1)$ \\
\hline$C(12)$ & $-197(1)$ & $5920(2)$ & $-818(1)$ & $65(1)$ \\
\hline$C(13)$ & $-72(2)$ & $4921(2)$ & $-1196(2)$ & $83(1)$ \\
\hline$C(14)$ & $1590(1)$ & $8131(1)$ & $2051(1)$ & $42(1)$ \\
\hline$C(15)$ & $779(1)$ & $8515(1)$ & $2007(1)$ & $50(1)$ \\
\hline$C(16)$ & $133(1)$ & $7969(1)$ & $2312(1)$ & $56(1)$ \\
\hline$C(17)$ & $266(1)$ & $7044(1)$ & $2670(1)$ & $55(1)$ \\
\hline$C(18)$ & $1075(1)$ & $6680(2)$ & $2717(1)$ & $69(1)$ \\
\hline$C(19)$ & $1742(1)$ & $7211(2)$ & $2416(1)$ & $61(1)$ \\
\hline$C(20)$ & $-444(2)$ & $6410(2)$ & $2990(1)$ & $80(1)$ \\
\hline
\end{tabular}


Table 3. Bond lengths [A] and angles [deg] for cd 24132.

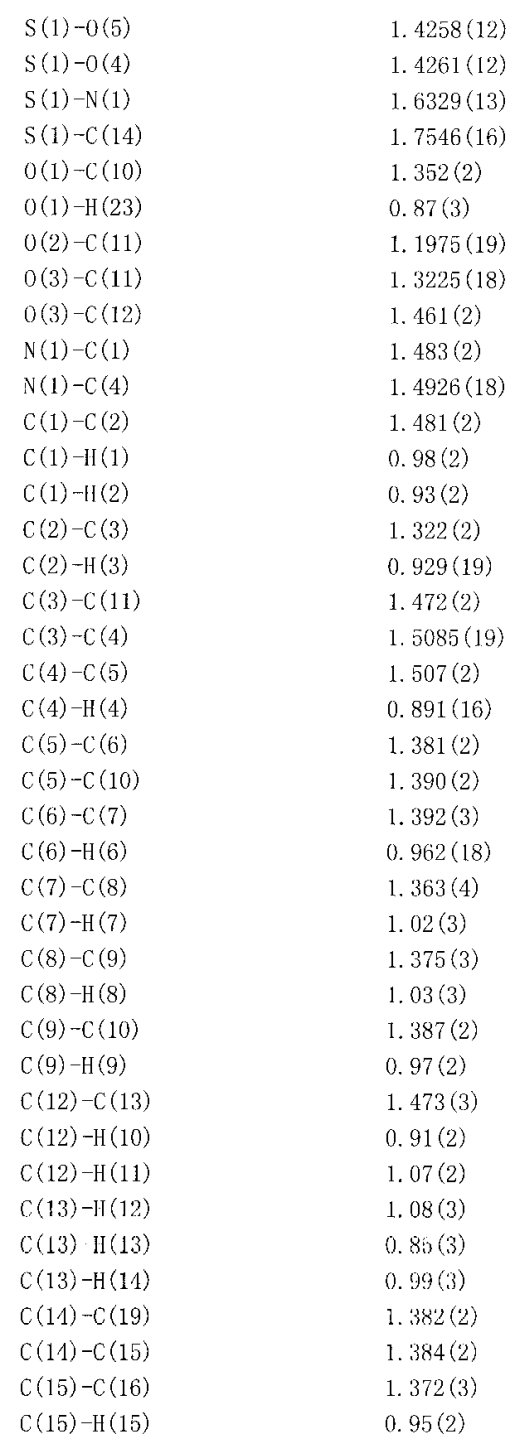

$\mathrm{S} 21$ 


$$
\|\|
$$




\begin{tabular}{|c|c|}
\hline$C(5)-C(6)-H(6)$ & $121.3(11)$ \\
\hline $\mathrm{C}(7)-\mathrm{C}(6)-\mathrm{H}(6)$ & $117.9(11)$ \\
\hline$C(8)-C(7)-C(6)$ & 119. 4(2) \\
\hline $\mathrm{C}(8)-\mathrm{C}(7)-\mathrm{H}(7)$ & $121.8(11)$ \\
\hline$C(6)-C(7)-I I(7)$ & $118.6(14)$ \\
\hline$C(7)-C(8)-C(9)$ & $121.02(19)$ \\
\hline $\mathrm{C}(7)-\mathrm{C}(8)-\mathrm{H}(8)$ & $130.1(16)$ \\
\hline$C(9)-C(8)-H(8)$ & $108.8(16)$ \\
\hline$C(8)-C(9)-C(10)$ & $119.6(2)$ \\
\hline $\mathrm{C}(8)-\mathrm{C}(9)-\mathrm{H}(9)$ & $120.7(14)$ \\
\hline $\mathrm{C}(10)-\mathrm{C}(9)-\mathrm{H}(9)$ & $119.7(14)$ \\
\hline $0(1)-C(10)-C(9)$ & $122.16(16)$ \\
\hline $0(1)-\mathrm{C}(10)-\mathrm{C}(5)$ & $117.46(13)$ \\
\hline$C(9)-C(10)-C(5)$ & $120.36(17)$ \\
\hline $0(2)-C(11)-0(3)$ & $123.41(15)$ \\
\hline$O(2)-\mathrm{C}(11)-\mathrm{C}(3)$ & $123.27(14)$ \\
\hline$O(3)-C(11)-C(3)$ & $113.28(13)$ \\
\hline$O(3)-C(12)-C(13)$ & $107.60(19)$ \\
\hline $\mathrm{O}(3)-\mathrm{C}(12)-\mathrm{H}(10)$ & $108.4(15)$ \\
\hline $\mathrm{C}(13)-\mathrm{C}(12)-\mathrm{H}(10)$ & $112.7(15)$ \\
\hline $\mathrm{O}(3)-\mathrm{C}(12)-\mathrm{H}(11)$ & $104.2(12)$ \\
\hline $\mathrm{C}(13)-\mathrm{C}(12)-\mathrm{H}(11)$ & $113.9(13)$ \\
\hline$H(10)-C(12)-\|(11)$ & $110(2)$ \\
\hline $\mathrm{C}(12)-\mathrm{C}(13)-\mathrm{H}(12)$ & $105.3(16)$ \\
\hline $\mathrm{C}(12)-\mathrm{C}(13)-\mathrm{H}(13)$ & $110.0(18)$ \\
\hline $\mathrm{H}(12)-\mathrm{C}(13)-\mathrm{H}(13)$ & $107(3)$ \\
\hline $\mathrm{C}(12)-\mathrm{C}(13)-\mathrm{H}(14)$ & $114.3(18)$ \\
\hline $\mathrm{II}(12)-\mathrm{C}(13)-\mathrm{H}(14)$ & $109(2)$ \\
\hline $\mathrm{II}(13)-\mathrm{C}(13)-\mathrm{H}(14)$ & $111(2)$ \\
\hline$C(19)-C(14)-C(15)$ & $119.42(15)$ \\
\hline$C(19)-C(14)-S(1)$ & $120.35(13)$ \\
\hline$C(15)-C(14)-S(1)$ & $120.14\langle 12\rangle$ \\
\hline$C(16)-C(15)-C(14)$ & $119.96(16)$ \\
\hline$C(16)-C(15)-H(15)$ & $120.1(12)$ \\
\hline$C(14)-C(15)-H(15)$ & $119.5(12)$ \\
\hline$C(15)-C(16)-C(17)$ & $121.67(18)$ \\
\hline$C(15)-C(16)-I I(16)$ & $120.0(13)$ \\
\hline$C(17)-C(16)-H(16)$ & $118.1(13)$ \\
\hline$C(18)-C(17)-C(16)$ & $117.75(17)$ \\
\hline$C(18)-C(17)-C(20)$ & $120.73(18)$ \\
\hline$c(16)-c(17)-c(20)$ & $121.52(19)$ \\
\hline$C(17)-c(18)-c(19)$ & $122.01(17)$ \\
\hline$C(17)-C(18)-H(18)$ & $115.6(13)$ \\
\hline$C(19)-C(18)-H(18)$ & $122.3(13)$ \\
\hline
\end{tabular}




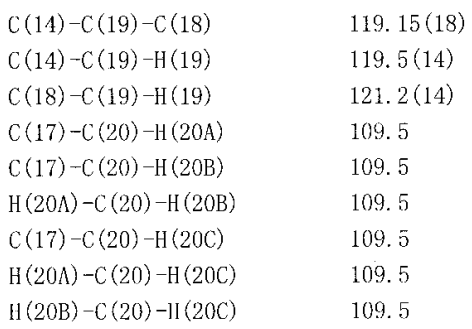

Symmetry transformations used to generate equivalent atoms:

S24 
Table 4. Anisotropic displacement parameters $\left(A^{\wedge} 2 \times 10^{\wedge} 3\right)$ for cd24432. The anisotropic displacement factor exponent takes the form: $-2 \mathrm{pi}^{\wedge} 2\left[\mathrm{~h}^{\wedge} 2 \mathrm{a} *^{\wedge} 2 \mathrm{v11}+\ldots+2 \mathrm{~h} \mathrm{k}\right.$ a* b* U12]

\begin{tabular}{lrrrrrr}
\hline & & & & & \\
& $\mathrm{V} 11$ & $\mathrm{U} 22$ & $\mathrm{U} 33$ & $\mathrm{U} 23$ & $\mathrm{U} 13$ & $\mathrm{U} 12$ \\
& & & & & & \\
\hline & & & & & \\
$\mathrm{S}(1)$ & $43(1)$ & $39(1)$ & $41(1)$ & $-5(1)$ & $-2(1)$ & $-5(1)$ \\
$0(1)$ & $57(1)$ & $13(1)$ & $72(1)$ & $2(1)$ & $0(1)$ & $14(1)$ \\
$\mathrm{O}(2)$ & $48(1)$ & $45(1)$ & $101(1)$ & $-9(1)$ & $-22(1)$ & $11(1)$ \\
$\mathrm{O}(3)$ & $50(1)$ & $40(1)$ & $72(1)$ & $-13(1)$ & $-17(1)$ & $1(1)$ \\
$0(4)$ & $67(1)$ & $35(1)$ & $59(1)$ & $-7(1)$ & $5(1)$ & $-8(1)$ \\
$0(5)$ & $46(1)$ & $73(1)$ & $56(1)$ & $-6(1)$ & $-13(1)$ & $-6(1)$ \\
$\mathrm{N}(1)$ & $37(1)$ & $40(1)$ & $41(1)$ & $-6(1)$ & $-3(1)$ & $5(1)$ \\
$\mathrm{C}(1)$ & $16(1)$ & $45(1)$ & $62(1)$ & $-11(1)$ & $-11(1)$ & $14(1)$ \\
$\mathrm{C}(2)$ & $44(1)$ & $38(1)$ & $57(1)$ & $-10(1)$ & $-4(1)$ & $7(1)$ \\
$\mathrm{C}(3)$ & $39(1)$ & $33(1)$ & $46(1)$ & $-4(1)$ & $0(1)$ & $1(1)$ \\
$\mathrm{C}(4)$ & $33(1)$ & $33(1)$ & $40(1)$ & $-3(1)$ & $0(1)$ & $3(1)$ \\
$\mathrm{C}(5)$ & $41(1)$ & $38(1)$ & $40(1)$ & $-4(1)$ & $-4(1)$ & $-8(1)$ \\
$\mathrm{C}(6)$ & $59(1)$ & $61(1)$ & $48(1)$ & $-6(1)$ & $7(1)$ & $-7(1)$ \\
$\mathrm{C}(7)$ & $83(2)$ & $90(2)$ & $48(1)$ & $-4(1)$ & $14(1)$ & $-31(1)$ \\
$\mathrm{C}(8)$ & $103(2)$ & $73(1)$ & $51(1)$ & $15(1)$ & $-11(1)$ & $-32(1)$ \\
$\mathrm{C}(9)$ & $81(1)$ & $50(1)$ & $58(1)$ & $12(1)$ & $-19(1)$ & $-14(1)$ \\
$\mathrm{C}(10)$ & $50(1)$ & $36(1)$ & $17(1)$ & $0(1)$ & $-13(1)$ & $-8(1)$ \\
$\mathrm{C}(11)$ & $43(1)$ & $33(1)$ & $52(1)$ & $1(1)$ & $-5(1)$ & $0(1)$ \\
$\mathrm{C}(12)$ & $63(1)$ & $57(1)$ & $76(1)$ & $-8(1)$ & $-26(1)$ & $-8(1)$ \\
$\mathrm{C}(13)$ & $94(2)$ & $71(2)$ & $84(2)$ & $-23(1)$ & $-24(2)$ & $-18(1)$ \\
$\mathrm{C}(14)$ & $49(1)$ & $39(1)$ & $39(1)$ & $-1(1)$ & $1(1)$ & $-1(1)$ \\
$\mathrm{C}(15)$ & $56(1)$ & $41(1)$ & $54(1)$ & $4(1)$ & $14(1)$ & $11(1)$ \\
$\mathrm{C}(16)$ & $51(1)$ & $55(1)$ & $61(1)$ & $2(1)$ & $17(1)$ & $9(1)$ \\
$\mathrm{C}(17)$ & $65(1)$ & $47(1)$ & $52(1)$ & $-4(1)$ & $13(1)$ & $-7(1)$ \\
$\mathrm{C}(18)$ & $78(1)$ & $53(1)$ & $74(1)$ & $26(1)$ & $2(1)$ & $0(1)$ \\
$\mathrm{C}(19)$ & $54(1)$ & $60(1)$ & $71(1)$ & $22(1)$ & $-7(1)$ & $4(1)$ \\
$\mathrm{C}(20)$ & $92(2)$ & $62(1)$ & $87(2)$ & $1(1)$ & $33(1)$ & $-18(1)$ \\
& & & & & & \\
\hline
\end{tabular}


Table 5. llydrogen coordinates $\left(x 10^{\wedge} 4\right)$ and isotropjc displacement parameters $\left(A^{\prime} 2 \times 10^{\wedge} 3\right)$ for cd24432.

\begin{tabular}{|c|c|c|c|c|}
\hline & $x$ & $\mathrm{y}$ & $z$ & $\mathrm{U}(\mathrm{eq})$ \\
\hline $\mathrm{H}(20 \mathrm{~A})$ & -764 & 6105 & 2628 & 121 \\
\hline $\mathrm{H}(20 \mathrm{~B})$ & -798 & 6913 & 3247 & 121 \\
\hline $\mathrm{H}(20 \mathrm{C})$ & -224 & 5914 & 3302 & 121 \\
\hline $\mathrm{H}(1)$ & $3236(13)$ & $7151(15)$ & $499(10)$ & $57(5)$ \\
\hline $\mathrm{H}(2)$ & $2794(13)$ & $6832(16)$ & $1170(11)$ & $62(5)$ \\
\hline$H(3)$ & $2028(11)$ & $5955(15)$ & $227(9)$ & $50(5)$ \\
\hline$H(4)$ & $1243(10)$ & $8748(11)$ & $639(8)$ & $34(4)$ \\
\hline$H(6)$ & $2762(11)$ & $8463(15)$ & $-694(9)$ & $49(5)$ \\
\hline$H(7)$ & $3018(16)$ & $9694(19)$ & $-1574(13)$ & $94(8)$ \\
\hline II $(8)$ & $2038(18)$ & $11290(20)$ & $-1623(14)$ & $106(9)$ \\
\hline$H(9)$ & $1056(15)$ & $11460(18)$ & $-817(12)$ & $87(7)$ \\
\hline$H(10)$ & $-360(14)$ & $6453(18)$ & $-1105(13)$ & $81(7)$ \\
\hline $\mathrm{H}(11)$ & $-622(15)$ & $5863(19)$ & $-387(12)$ & $82(7)$ \\
\hline $\mathrm{H}(12)$ & $370(20)$ & $5100(20)$ & $-1604(18)$ & $133(11)$ \\
\hline$H(13)$ & $-523(18)$ & $4740(20)$ & $-1397(15)$ & $97(8)$ \\
\hline$H(14)$ & $151(19)$ & $4340(30)$ & $-903(15)$ & $119(10)$ \\
\hline$H(15)$ & $681(12)$ & $9175(16)$ & $1793(9)$ & $58(5)$ \\
\hline JI (16) & $-384(13)$ & $8245(16)$ & $2317(11)$ & $66(6)$ \\
\hline$H(18)$ & $1139(13)$ & $6064(16)$ & $2944(10)$ & $64(5)$ \\
\hline$H(19)$ & $2312(14)$ & $6917(18)$ & $2425(12)$ & $78(6)$ \\
\hline II (23) & $449(16)$ & $10870(20)$ & $232(12)$ & $91(8)$ \\
\hline
\end{tabular}


Table 6. Torsion angles [deg] for cd24432.

$\begin{array}{lc}O(5)-S(1)-N(1)-C(1) & 46.06(13) \\ O(4)-S(1)-N(1)-C(1) & 175.55(12) \\ C(14)-S(1)-N(1)-C(1) & -69.47(13) \\ O(5)-S(1)-N(1)-C(4) & 178.97(10) \\ O(4)-S(1)-N(1)-C(1) & -51.55(12) \\ C(14)-S(1)-N(1)-C(4) & 63.44(12) \\ C(4)-N(1)-C(1)-C(2) & -6.03(18) \\ S(1)-N(1)-C(1)-C(2) & 129.29(13) \\ N(1)-C(1)-C(2)-C(3) & 2.7(2) \\ C(1)-C(2)-C(3)-C(11) & -172.96(16) \\ C(1)-C(2)-C(3)-C(4) & 1.7(2) \\ C(1)-N(1)-C(4)-C(5) & -115.60(14) \\ S(1)-N(1)-C(4)-C(5) & 108.45(12) \\ C(1)-N(1)-C(4)-C(3) & 6.79(16) \\ S(1)-N(1)-C(4)-C(3) & -129.16(11) \\ C(2)-C(3)-C(4)-N(1) & -5.22(17) \\ C(11)-C(3)-C(1)-N(1) & 169.87(13) \\ C(2)-C(3)-C(4)-C(5) & 116.30(15) \\ C(11)-C(3)-C(4)-C(5) & -68.60(18) \\ N(1)-C(4)-C(5)-C(6) & 57.95(19) \\ C(3)-C(4)-C(5)-C(6) & -57.24(19) \\ N(1)-C(4)-C(5)-C(10) & -121.92(14) \\ C(3)-C(4)-C(5)-C(10) & 122.89(15) \\ C(10)-C(5)-C(6)-C(7) & -0.7(3) \\ C(4)-C(5)-C(6)-C(7) & 179.41(17) \\ C(5)-C(6)-C(7)-C(8) & -1.1(3) \\ C(6)-C(7)-C(8)-C(9) & 1.0(3) \\ C(7)-C(8)-C(9)-C(10) & 0.9(3) \\ C(8)-C(9)-C(10)-0(1) & 175.37(17) \\ C(8)-C(9)-C(10)-C(5) & -2.8(3) \\ C(6)-C(5)-C(10)-0(1) & -175.58(15) \\ C(4)-C(5)-C(10)-0(1) & 4.3(2) \\ C(6)-C(5)-C(10)-C(9) & 2.7(2) \\ C(4)-C(5)-C(10)-C(9) & -177.44(14) \\ C(12)-0(3)-C(11)-0(2) & -1.8(3) \\ C(12)-0(3)-C(11)-C(3) & 176.28(16) \\ C(2)-C(3)-C(11)-0(2) & 163.17(18) \\ C(4)-C(3)-C(11)-0(2) & -11.1(2) \\ C(2)-C(3)-C(11)-O(3) & -14.9(2) \\ C(1)-C(3)-C(11)-0(3) & 170.82(13) \\ & \\ C & \end{array}$




$$
\text { | }
$$


Table 7. Hydrogen bonds for cd24432 [A and deg.].

D-H. . A

$d(D-H)$

$d(H \ldots A) \quad d(D \ldots A)$

$<(\mathrm{DHA})$

S29 


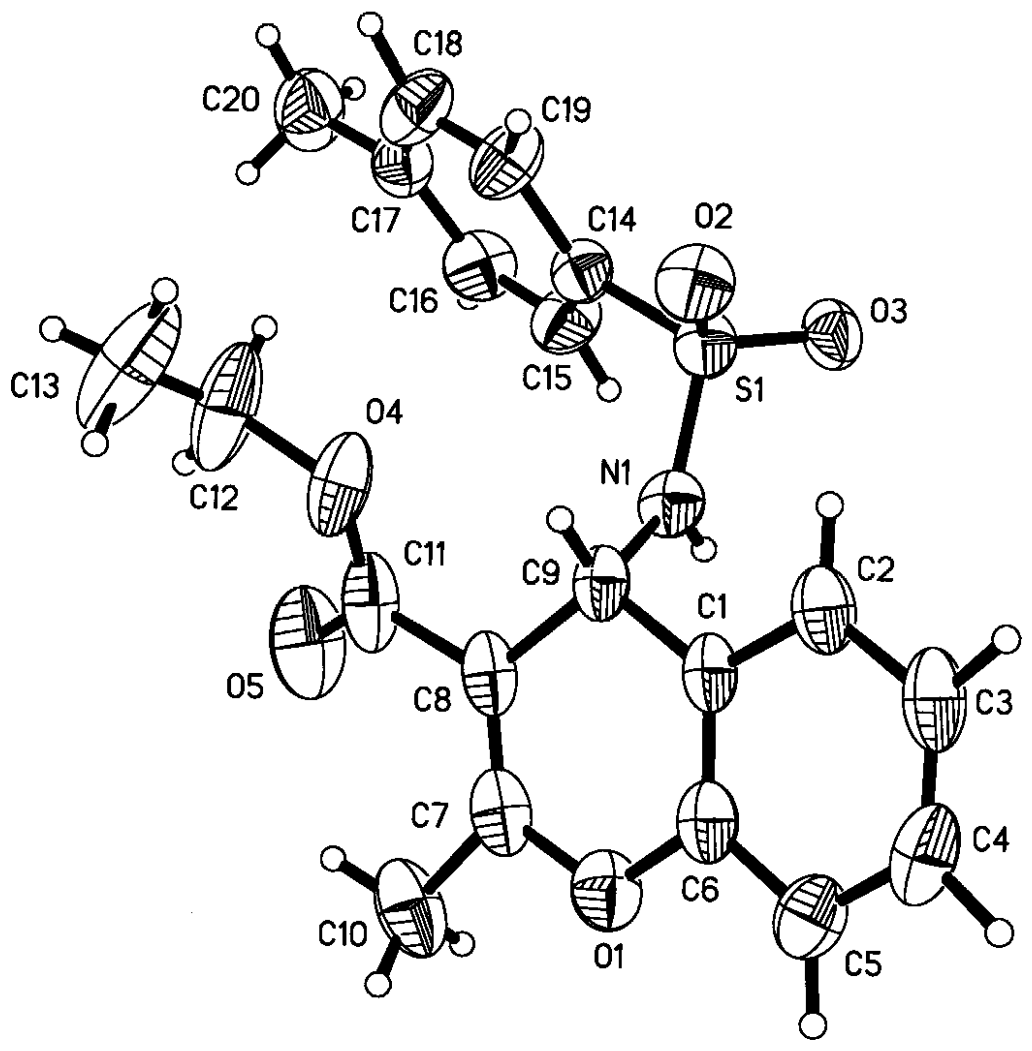

Figure 1. The ORTEP draw of $\mathbf{4 a}$.

The crystal data of $\mathbf{4 a}$ has been deposited in CCDC with number 266105. Empirical Formula: $\mathrm{C}_{20} \mathrm{H}_{21} \mathrm{NO}_{5} \mathrm{~S}$; Formula Weight: 387.44; Temperature: 293(2)K; Crystal Dimensions: $0.516 \mathrm{x}$ 0.354 x $0.220 \mathrm{~mm}$; Crystal System: Monoclinic; Space group: P2(1)/c; Unit cell dimensions: $a$

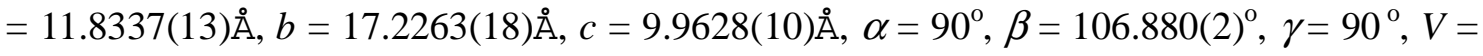
1943.4(4) $\AA^{3} ; Z=4 ; D_{\text {calc. }}=1.324 \mathrm{mg} / \mathrm{m}^{3} ; F_{000}=816$; Final R induces $[\mathrm{I}>2 \sigma(\mathrm{I})]: R_{1}=0.0642$, $R_{2}=0.1617$ 
'lable 1 . Crystal data and structure refinement for cdzb44.

Identification code

Empirical formula

Formula weight

Temperature

wavelength

Crystal system, space group

Unit cell dimensions

Volume

2, Calculated density

Absorption coefficient

E(000)

Crystal size

Theta range for data collection

Limiting indices

Reflections collected / unique

Completeness to theta $=26.50$

Absorption correction

Max, and min. transmission

Refinement method

Data / restraints / parameters

Goodness-offit on $\mathrm{F}^{\wedge} 2$

Final $R$ indices [ $I>2$ sigma(I)]

$\mathrm{R}$ indices (all data)

Largest diff. peak and hole $\operatorname{cd} 2544$

C20 H21 N $05 \mathrm{~S}$

387.44

$293(2)$ K

$0.71073 \mathrm{~A}$

Monoclinic, $\mathrm{P} 2(1) / \mathrm{C}$

$a=11.8337(13) \mathrm{A}$ alpha $=90 \mathrm{deg}$.

$\mathrm{b}=17.2263(18) \mathrm{A} \quad$ beta $=106.880(2) \mathrm{deg}$.

$c=9.9628(10) \mathrm{A}$ gamma $=90 \mathrm{deg}$.

$1943.4(4) A^{\wedge} 3$

4. $1.324 \mathrm{Mg} / \mathrm{m}^{\wedge} 3$

$0.197 \mathrm{~mm}^{\wedge}-1$

816

$0.516 \times 0.354 \times 0.220 \mathrm{~mm}$

1.80 to $26.50 \mathrm{deg}$.

$-14<=h<=13,-17<=k<=21,-12<=1<=12$

$10931 / 4005[R($ int $)=0.1340]$

$99.6 \%$

Empirical

1.00000 and 0.60697

Full-matrix least-squares on $F^{\wedge} 2$

$4005 / 0 / 287$

0.921

$\mathrm{R} 1=0.0642, \mathrm{wR} 2=0.1617$

$\mathrm{R} 1=0.1008, \omega \mathrm{WR} 2=0.1801$

0.293 and -0.226 e. $A^{\wedge}-3$ 
Table 2. Atomic coordinates $\left(x^{10} 4\right)$ and equivalent isotropic displacement parameters ( $A^{\wedge} 2 \times 10^{\wedge} 3$ ) for $c d 2544$.

$U(\mathrm{eq})$ is defined as one third of the trace of the orthogonalized Uij tensor.

\begin{tabular}{|c|c|c|c|c|}
\hline & x & $y$ & z & $0(e q)$ \\
\hline$S(1)$ & $3938(1)$ & $10465(1)$ & $2851(1)$ & $66(1)$ \\
\hline$o(1)$ & $1437(2)$ & $9749(1)$ & $6393(2)$ & $82(1)$ \\
\hline $0(2)$ & $3180(2)$ & $11055(1)$ & $2064(2)$ & $83(1)$ \\
\hline $0(3)$ & $5127(2)$ & $10664(1)$ & $3634(2)$ & $77(1)$ \\
\hline $0(4)$ & $1241(2)$ & $9177(2)$ & $1807(3)$ & $104(1)$ \\
\hline $0(5)$ & $1512(2)$ & $8143(2)$ & $3182(3)$ & $131(1)$ \\
\hline $\mathrm{N}(1)$ & $3344\langle 2\rangle$ & $10112(2)$ & $3948(3)$ & $65(1)$ \\
\hline$C(1)$ & $2023(2)$ & $10759(2)$ & $5057(3)$ & $60(1)$ \\
\hline$C(2)$ & $2295(3)$ & $11539(2)$ & $4989(4)$ & $78(1)$ \\
\hline$C(3)$ & $2241(3)$ & $12043(2)$ & $6047(5)$ & $93(1)$ \\
\hline$C(4)$ & $1889(3)$ & $11778(3)$ & $7145(5)$ & $98(1)$ \\
\hline$c(5)$ & $1622(3)$ & $11008(2)$ & $7257(4)$ & $83(1)$ \\
\hline$c(6)$ & $1702(2)$ & $10514(2)$ & $6200(3)$ & $67(1)$ \\
\hline$c(7)$ & $1395(2)$ & $9224(2)$ & $5356(4)$ & $75(1)$ \\
\hline$C(8)$ & $1628(2)$ & $9413(2)$ & $4174(4)$ & $67(1)$ \\
\hline$C(9)$ & $2105(2)$ & $10199(2)$ & $3951(3)$ & $61(1)$ \\
\hline$C(10)$ & $1025(3)$ & $8462(2)$ & $5790(4)$ & $106(1)$ \\
\hline$C(11)$ & $1474(3)$ & $8836(2)$ & $3044(5)$ & $89(1)$ \\
\hline$c(12)$ & $1054(4)$ & $8678(3)$ & $591(5)$ & $129(2)$ \\
\hline$c(13)$ & $108(5)$ & $8808(4)$ & $-406(5)$ & $126(3)$ \\
\hline$C(14)$ & $4043(2)$ & $9723(2)$ & $1693(3)$ & $65(1)$ \\
\hline$C(15)$ & $4633(3)$ & $9047(2)$ & $2215(4)$ & $83(1)$ \\
\hline$C(16)$ & $4734(3)$ & $8471(2)$ & $1319(4)$ & $90(1)$ \\
\hline$C(17)$ & $4257(3)$ & $8535(2)$ & $-117(4)$ & $81(1)$ \\
\hline$C(18)$ & $3698(3)$ & $9226(3)$ & $-622(4)$ & $98(1)$ \\
\hline$C(19)$ & $3592(3)$ & $9813(2)$ & $268(3)$ & $88(1)$ \\
\hline$C(20)$ & $4345(3)$ & $7885(3)$ & $-1091(4)$ & $116(1)$ \\
\hline
\end{tabular}


Table 3. Bond lengths [A] and ang]es [deg] for cd2544.

\begin{tabular}{|c|c|}
\hline$S(1)-O(2)$ & $1.4305(18)$ \\
\hline$S(1)-O(3)$ & $1.4381(18)$ \\
\hline$S(1)-N(1)$ & $1.583(3)$ \\
\hline$S(1)-C(14)$ & $1.750\langle 3\rangle$ \\
\hline$O(1)-C(7)$ & $1.363(4)$ \\
\hline$O(1)-C(6)$ & $1.381(3)$ \\
\hline $0(4)-C(11)$ & $1.321(4)$ \\
\hline$O(4)-C(12)$ & $1.450(4)$ \\
\hline $0(5)-C(11)$ & $1.200(4)$ \\
\hline$N(1)-C(9)$ & $1.474(4)$ \\
\hline $\mathrm{N}(1)-\mathrm{H}(1)$ & $0.77(3)$ \\
\hline$C(1)-C(6)$ & $1.367(4)$ \\
\hline$C(1)-C(2)$ & $1.387(4)$ \\
\hline$C(1)-C(9)$ & $1.490(4)$ \\
\hline$C(2)-C(3)$ & $1.382(5)$ \\
\hline $\mathrm{C}(2)-\mathrm{H}(2)$ & $0.95(3)$ \\
\hline$C(3)-C(4)$ & $1.358(6)$ \\
\hline $\mathrm{C}(3)-\mathrm{H}(3)$ & $0.95(3)$ \\
\hline$C(4)-C(5)$ & $1.374\{6\}$ \\
\hline $\mathrm{C}(4)-\mathrm{H}(4)$ & $1.02(4)$ \\
\hline$C(5)-c(6)$ & $1.379(5)$ \\
\hline$C(5)-H(5)$ & $0.97(3)$ \\
\hline$C(7)-C(8)$ & $1.326(4)$ \\
\hline$C(7)-C(10)$ & $1.488(4)$ \\
\hline$C(8)-C(11)$ & $1.474(4)$ \\
\hline$C(8)-C(9)$ & $1.507(4)$ \\
\hline $\mathrm{C}(9)-\mathrm{H}(9)$ & $0.87(3)$ \\
\hline $\mathrm{C}(10)-\mathrm{H}(10 \mathrm{~A})$ & 0.9600 \\
\hline $\mathrm{C}(10)-\mathrm{H}(10 \mathrm{~B})$ & 0.9600 \\
\hline $\mathrm{C}(10)-\mathrm{H}(10 \mathrm{C})$ & 0.9600 \\
\hline$C(12)-C(13)$ & $1.282\langle 5\rangle$ \\
\hline $\mathrm{C}(12)-\mathrm{H}(12 \mathrm{~A})$ & 0.9700 \\
\hline $\mathrm{C}(12)-\mathrm{H}(12 \mathrm{~B})$ & 0.9700 \\
\hline$C(13)-H(13 A)$ & 0.9600 \\
\hline$C(13)-H(13 B)$ & 0.9600 \\
\hline $\mathrm{C}(13)-\mathrm{H}(13 \mathrm{C})$ & 0.9600 \\
\hline$C(14)-C(19)$ & $1.372(4)$ \\
\hline$C(14)-C(15)$ & $1.379(4)$ \\
\hline$C(15)-C(16)$ & $1.363(5)$ \\
\hline$C(15)-H(15)$ & $0.92(3)$ \\
\hline$C(16)-C(17)$ & $1.381(5)$ \\
\hline$C(16)-H(16)$ & $0.94(4)$ \\
\hline$C(17)-C(18)$ & $1.384(5)$ \\
\hline$C(17)-C(20)$ & $1.505(5)$ \\
\hline$C(18)-C(19)$ & $1.375(5)$ \\
\hline $\mathrm{C}(18)-\mathrm{H}(18)$ & $0.96(3)$ \\
\hline$C(19)-H(19)$ & $1.09(4)$ \\
\hline$C(20)-H(20 A)$ & 0.9600 \\
\hline$C(20)-H(20 B)$ & 0.9600 \\
\hline$C(20)-H(20 C)$ & 0.9600 \\
\hline$O(2)-S(1)-O(3)$ & $119.12(13)$ \\
\hline$O(2)-S(1)-N(1)$ & $108.54(14)$ \\
\hline$O(3)-S(1)-N(1)$ & $106.10(12)$ \\
\hline $0(2)-5(1)-C(14)$ & $108.13(12)$ \\
\hline $0(3)-5(1)-c(14)$ & $106.67(14)$ \\
\hline$N(1)-S(1)-C(14)$ & $1.07 .81(14)$ \\
\hline$c(7)-0(1)-c(6)$ & $119.1(3)$ \\
\hline$C(11)-O(4)-C(12)$ & $117.1(3)$ \\
\hline$C(9)-\mathrm{N}(1)-\mathrm{S}(1)$ & $127.1(2)$ \\
\hline $\mathrm{C}(9)-\mathrm{N}(1)-\mathrm{H}(1)$ & $114(2)$ \\
\hline$S(1)-N(1)-H(1)$ & $118(2)$ \\
\hline$C(6)-C(1)-C(2)$ & $117.6(3)$ \\
\hline$C(6)-C(1)-C(9)$ & $120.6(3)$ \\
\hline$C(2)-C(1)-C(9)$ & $121.7(3)$ \\
\hline$C(3)-C(2)-C(1)$ & $120.6(4)$ \\
\hline $\mathrm{C}(3)-\mathrm{C}(2)-\mathrm{H}(2)$ & $1.24(2)$ \\
\hline$C(1)-C(2)-H(2)$ & $114(2)$ \\
\hline
\end{tabular}




$$
\begin{aligned}
& C(4)-C(3)-C(2) \\
& C(4)-C(3)-H(3) \\
& \mathrm{C}(2)-\mathrm{C}(3)-\mathrm{H}(3) \\
& C(3)-C(4)-C(5) \\
& C(3)-C(4)-H(4) \\
& \mathrm{C}(5)-\mathrm{C}(4)-\mathrm{H}(4) \\
& C(4)-C(5)-C(6) \\
& \text { C(4) }-C(5)-C(6) \\
& C(4)-C(5)-H(5) \\
& C(6)-C(5)-H(5) \\
& C(1)-C(6)-C(5) \\
& C(1)-C(6)-O(1) \\
& C(5)-C(6)-O(1) \\
& C(8)-C(7)-O(1) \\
& C(8)-C(7)-C(10) \\
& 0(1)-C(7)-C(10) \\
& \mathrm{C}(7)-\mathrm{C}(8)-\mathrm{C}(11) \\
& C(7)-C(8)-C(9) \\
& C(11)-C(8)-C(9) \\
& \mathrm{N}(1)-\mathrm{C}(9)-\mathrm{C}(1) \\
& \mathrm{N}(1)-\mathrm{C}(9)-\mathrm{C}(8) \\
& C(1)-C(9)-C(8) \\
& \mathrm{N}(1)-\mathrm{C}(9)-\mathrm{H}(9) \\
& \text { C(1) }-C(9)-H(9) \\
& \mathrm{C}(8)-\mathrm{C}(9)-\mathrm{H}(9) \\
& C(7)-C(10)-H(10 A) \\
& C(7)-C(10)-H(10 B) \\
& \mathrm{H}(10 \mathrm{~A})-\mathrm{C}(10)-\mathrm{H}(10 \mathrm{~B}) \\
& C(7)-C(10)-H(10 C) \\
& \mathrm{H}(10 \mathrm{~A})-\mathrm{C}(10)-\mathrm{H}(10 \mathrm{C}) \\
& \mathrm{H}(10 \mathrm{~B})-\mathrm{C}(10)-\mathrm{H}(10 \mathrm{C}) \\
& O(5)-C(11)-O(4) \\
& 0(5)-C(11)-C(8) \\
& O(4)-C(11)-C(8) \\
& C(13)-C(12)-O(4) \\
& C(13)-C(12)-H(12 A) \\
& \mathrm{O}(4)-\mathrm{C}(12)-\mathrm{H}(12 \mathrm{~A}) \\
& C(13)-C(12)-H(12 B) \\
& \text { O(4)-C(12)-H(12B) } \\
& \mathrm{H}(12 \mathrm{~A})-\mathrm{C}(12)-\mathrm{H}(12 \mathrm{~B}) \\
& \mathrm{C}(12)-\mathrm{C}(13)-\mathrm{H}(13 \mathrm{~A}) \\
& C(12)-C(13)-H(13 B) \\
& \mathrm{H}(13 \mathrm{~A})-\mathrm{C}(13)-\mathrm{H}(13 \mathrm{~B}) \\
& C(12)-C(13)-\mathrm{H}(13 \mathrm{C}) \\
& \mathrm{H}(13 \mathrm{~A})-\mathrm{C}(13)-\mathrm{H}(13 \mathrm{C}) \\
& \mathrm{H}(13 \mathrm{~B})-\mathrm{C}(13)-\mathrm{H}(13 \mathrm{C}) \\
& C(19)-C(14)-C(15) \\
& C(19)-C(14)-S(1) \\
& C(15)-C(14)-S(1) \\
& C(16)-C(15)-C(14) \\
& C(16)-C(15)-H(15) \\
& \mathrm{C}(14)-\mathrm{C}(15)-\mathrm{H}(15) \\
& C(15)-C(16)-C(17) \\
& C(15)-C(16)-H(16) \\
& \text { C(17) }-C(16) \\
& C(16)-C(17)-C(18) \\
& C(16)-C(17)-C(20) \\
& C(18)-C(17)-C(20) \\
& C(19)-C(18)-C(17) \\
& \mathrm{C}(19)-\mathrm{C}(18)-\mathrm{H}(18) \\
& C(1)-C(18)-H(18) \\
& C(14)-C(19)-C(18) \\
& \text { c(14)-C(19)-C(18) } \\
& C(14)-C(19)-H(19) \\
& C(18)-C(19)-H(19) \\
& C(17)-C(20)-H(20 A) \\
& C(17)-C(20)-H(20 E) \\
& \mathrm{H}(20 \mathrm{~A})-\mathrm{C}(2 \mathrm{O})-\mathrm{H}(2 \mathrm{OB}) \\
& \mathrm{C}(1.7)-\mathrm{C}(20)-\mathrm{H}(20 \mathrm{C}) \\
& \mathrm{H}(20 \mathrm{~A})-\mathrm{C}(20)-\mathrm{H}(20 \mathrm{C}) \\
& \mathrm{H}(20 \mathrm{~B})-\mathrm{C}(20)-\mathrm{H}(20 \mathrm{C})
\end{aligned}
$$

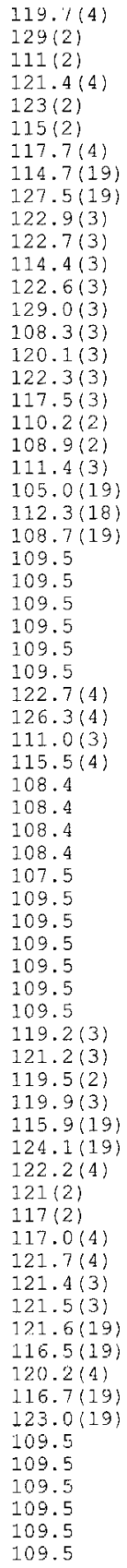

Symmetry transformations used to generate equivalent atoms: 
Table 4. Anisotropic displacement parameters $\left(A^{\wedge} 2 \times 10^{\wedge} 3\right.$ ) for cd2544. The anisotropic displacement factor exponent takes the form:

$-2 \mathrm{pi \wedge}\left[\mathrm{h}^{\wedge} 2 \mathrm{a}^{\star \wedge} 2011+\ldots+2 \mathrm{~h} k \mathrm{a}^{*} \mathrm{~b}^{*} \mathrm{Ul2}\right]$

\begin{tabular}{|c|c|c|c|c|c|c|}
\hline & U11 & $\mathrm{U} 22$ & 033 & $\mathrm{U} 23$ & 013 & $\mathrm{v} 12$ \\
\hline$S(1)$ & $68(1)$ & $55(1)$ & $64(1)$ & $12(1)$ & $1(1)$ & $5(1)$ \\
\hline$O(1)$ & $89(2)$ & $61(1)$ & $86(1)$ & $13(1)$ & $9(1)$ & $0(1)$ \\
\hline$O(2)$ & $96(2)$ & $59(1)$ & $80(1)$ & $24(1)$ & $3(1)$ & $21(1)$ \\
\hline $0(3)$ & $70(1)$ & $74(1)$ & $75(1)$ & $11(1)$ & $-1(1)$ & $-14(1)$ \\
\hline $0(4)$ & $98(2)$ & $81(2)$ & $106(2)$ & $-41(2)$ & $-14(1)$ & $17(1)$ \\
\hline $0(5)$ & $136(2)$ & $48(2)$ & $195(3)$ & $-35(2)$ & $26(2)$ & $6(1)$ \\
\hline $\mathrm{N}(1)$ & $61(2)$ & $66(2)$ & $59(1)$ & $13(1)$ & $0(1)$ & $13(1)$ \\
\hline$C\{1\}$ & $52(2)$ & $43(2)$ & $72(2)$ & $-4(1)$ & $-3(1)$ & 1 (1) \\
\hline$C(2)$ & $76(2)$ & $50(2)$ & $99(2)$ & $-8(2)$ & $13(2)$ & $-2(1)$ \\
\hline$C(3)$ & $94(3)$ & $49(2)$ & $122(3)$ & $-21(2)$ & $8(2)$ & $-6(2)$ \\
\hline$C(A)$ & $95(3)$ & $89(3)$ & $96(3)$ & $-37(3)$ & $5(2)$ & $6(2)$ \\
\hline$C(5)$ & $80(2)$ & $84(3)$ & $77(2)$ & $-9(2)$ & $9(2)$ & $9(2)$ \\
\hline$C(6)$ & $55(2)$ & $55(2)$ & $77(2)$ & $-2(2)$ & $-2(1)$ & $6(1)$ \\
\hline$C(7)$ & $59(2)$ & $48(2)$ & $101(2)$ & $5(2)$ & $-4(2)$ & $5(1)$ \\
\hline$C(8)$ & $50(2)$ & $41(2)$ & $95(2)$ & $-5(2)$ & $-3(2)$ & $5(1)$ \\
\hline$C(9)$ & $53(2)$ & $48(2)$ & $67(2)$ & $-1(1)$ & $-4(1)$ & $6(1)$ \\
\hline$C(10)$ & $93(2)$ & $53(2)$ & $152(3)$ & $25(2)$ & $5(2)$ & $-8(2)$ \\
\hline$C(11)$ & $55(2)$ & $60(2)$ & $134(3)$ & $-31(2)$ & $-3(2)$ & $8(1)$ \\
\hline$c(12)$ & $121(3\}$ & $137(4)$ & $150(4)$ & $-98(3)$ & $-20(3)$ & $33(3)$ \\
\hline$C(13)$ & $173(5)$ & $240(7)$ & $102(3)$ & $-76(4)$ & $-29(3)$ & $87(5)$ \\
\hline$C\{14\}$ & $58(2)$ & $66(2)$ & $63(2)$ & $10(1)$ & $3(1)$ & $5(1)$ \\
\hline$C(15)$ & $106(3)$ & $67(2)$ & $68(2)$ & $12(2)$ & $10(2)$ & $14(2)$ \\
\hline$C(16)$ & $103(3)$ & $68(2)$ & $99(3)$ & $7(2)$ & $29(2)$ & $15(2)$ \\
\hline$C(17)$ & $72(2)$ & $87(3)$ & $84(2)$ & $-13(2)$ & $24(2)$ & $-8(2)$ \\
\hline$c(18)$ & $87(2)$ & $128(4)$ & $65(2)$ & $-6(2)$ & $2(2)$ & $11(2)$ \\
\hline$C(19)$ & $88(2)$ & $97(3)$ & $64(2)$ & $3(2)$ & $-2(2)$ & $22(2)$ \\
\hline$c(20)$ & $111(3)$ & $117(3)$ & $125(3)$ & $-38(3)$ & $46(2)$ & $-13(2)$ \\
\hline
\end{tabular}


Table 5. Hydrogen coordinates $\left(x 10^{n} 4\right)$ and isotropic displacement parameters $\left(A^{\wedge} 2 \times 10^{\wedge} 3\right)$ for cd2544.

\begin{tabular}{lcccc} 
& & & & \\
& $x$ & & & \\
& & & & \\
& & & & \\
H $(10 A)$ & 893 & 8101 & 5025 & 128 \\
$\mathrm{H}(10 \mathrm{~B})$ & 309 & 8525 & 6047 & 128 \\
$\mathrm{H}(10 \mathrm{C})$ & 1634 & 8266 & 6579 & 128 \\
$\mathrm{H}(12 \mathrm{~A})$ & 1724 & 8732 & 222 & 138 \\
$\mathrm{H}(12 \mathrm{~B})$ & 1038 & 8143 & 893 & 138 \\
$\mathrm{H}(13 \mathrm{~A})$ & -554 & 8825 & -33 & 139 \\
$\mathrm{H}(13 \mathrm{~B})$ & -7 & 8400 & -1089 & 139 \\
$\mathrm{H}(13 \mathrm{C})$ & 177 & 9296 & -841 & 139 \\
$\mathrm{H}(20 \mathrm{~A})$ & 3924 & 7441 & -909 & 133 \\
$\mathrm{H}(20 \mathrm{~B})$ & 5160 & 7752 & -942 & 133 \\
$\mathrm{H}(20 \mathrm{C})$ & 4008 & 8049 & -2045 & 133 \\
$\mathrm{H}(1)$ & $3750(20)$ & $9938(15)$ & $4630(30)$ & $53(8)$ \\
$\mathrm{H}(2)$ & $2630(30)$ & $11665(19)$ & $4260(30)$ & $89(11)$ \\
$\mathrm{H}(3)$ & $2400(30)$ & $12560(20)$ & $5820(30)$ & $96(11)$ \\
$\mathrm{H}(4)$ & $1740(30)$ & $12130(20)$ & $7900(40)$ & $109(11)$ \\
$\mathrm{H}(5)$ & $1360(30)$ & $10883(18)$ & $8070(30)$ & $80(10)$ \\
$\mathrm{H}(9)$ & $1730(20)$ & $10359(16)$ & $3120(30)$ & $69(9)$ \\
$\mathrm{H}(15)$ & $4940(20)$ & $8941(17)$ & $3150(30)$ & $81(9)$ \\
$\mathrm{H}(16)$ & $5110(30)$ & $8000(20)$ & $1660(40)$ & $117(13)$ \\
$\mathrm{H}(18)$ & $3480(20)$ & $9299(17)$ & $-1620(30)$ & $78(9)$ \\
$\mathrm{H}(19)$ & $3100(30)$ & $10350(20)$ & $-100(40)$ & $120(12)$ \\
& & & & \\
\hline
\end{tabular}


Table 6. Torsion angles [deg] for cd2544.

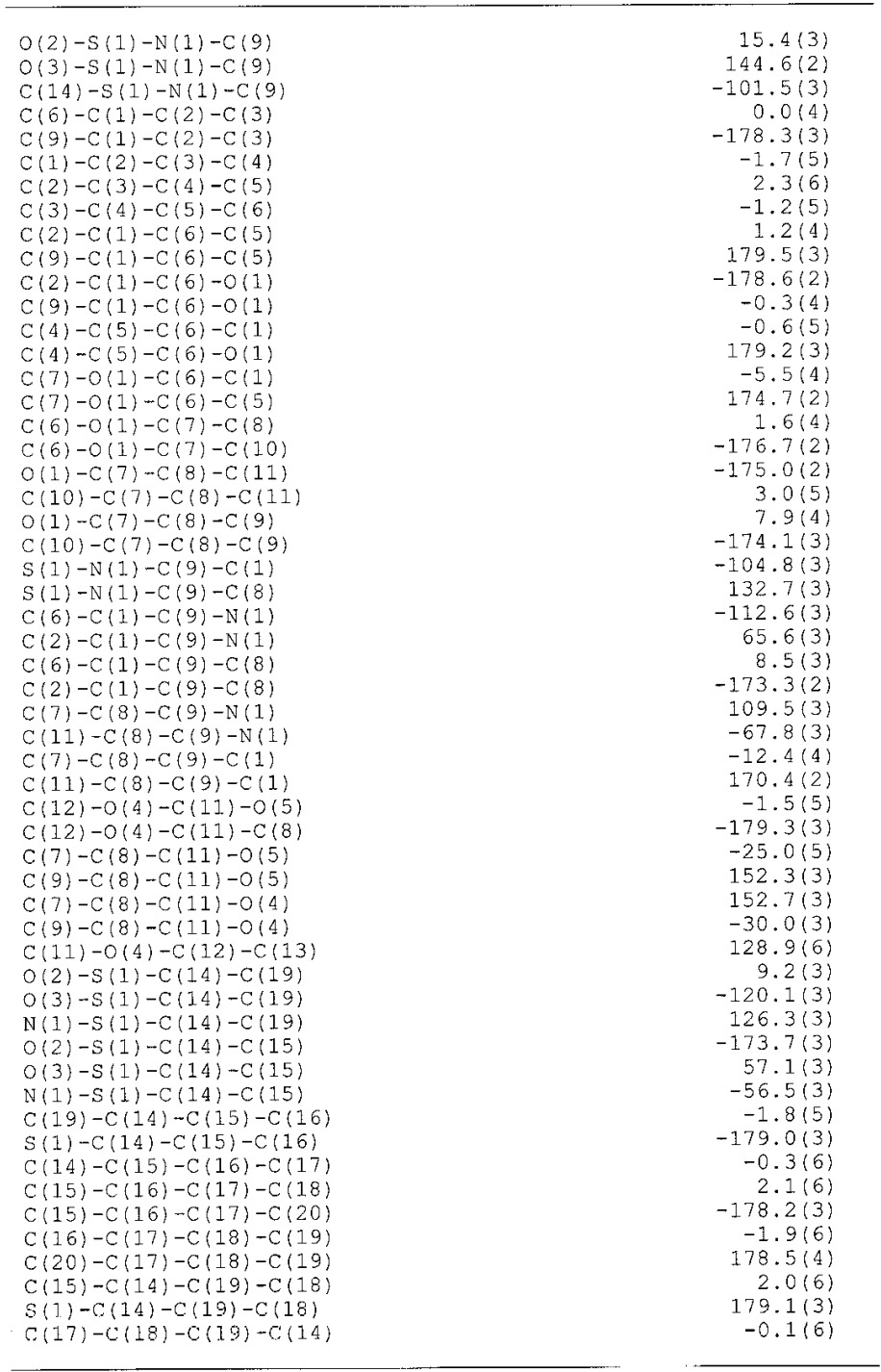

Symetry Lransformations used to generate equivalent atoms: 
'l'able 7. Hydrogen bonds for cd2544 [A and deg.].

\begin{tabular}{lllll}
\hline$D \sim H \ldots A$ & $d(D-H)$ & $d(H \ldots A)$ & $d(D \ldots A)$ & $<(D H A)$ \\
$N(1)-H(1) \ldots O(3) \# 1$ & $0.77(3)$ & $2.12(3)$ & $2.886(3)$ & $173(3)$ \\
\hline
\end{tabular}

Symmetry transformations used to generate equivalent atoms:

$\# 1-x+1,-y+2,-z+1$ 
The 2D NOESY spectrum of 2-(1-methyl-3-oxo-but-1-enyl)benzaldehyde 13a:

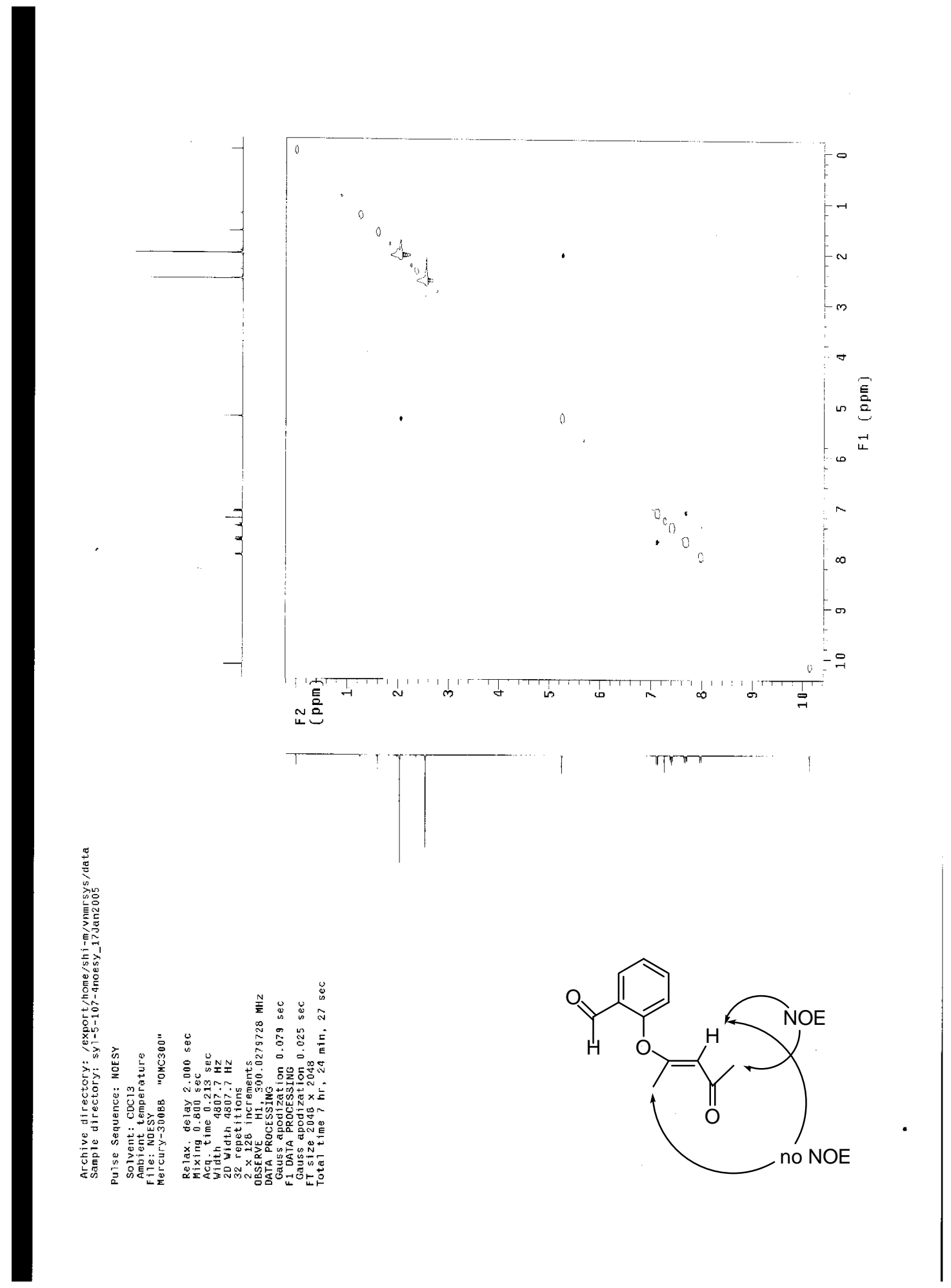


The 2D NOESY spectrum of 3-(2-formyl-phenyl)-but-2-enoic acid ethyl ester (13b):

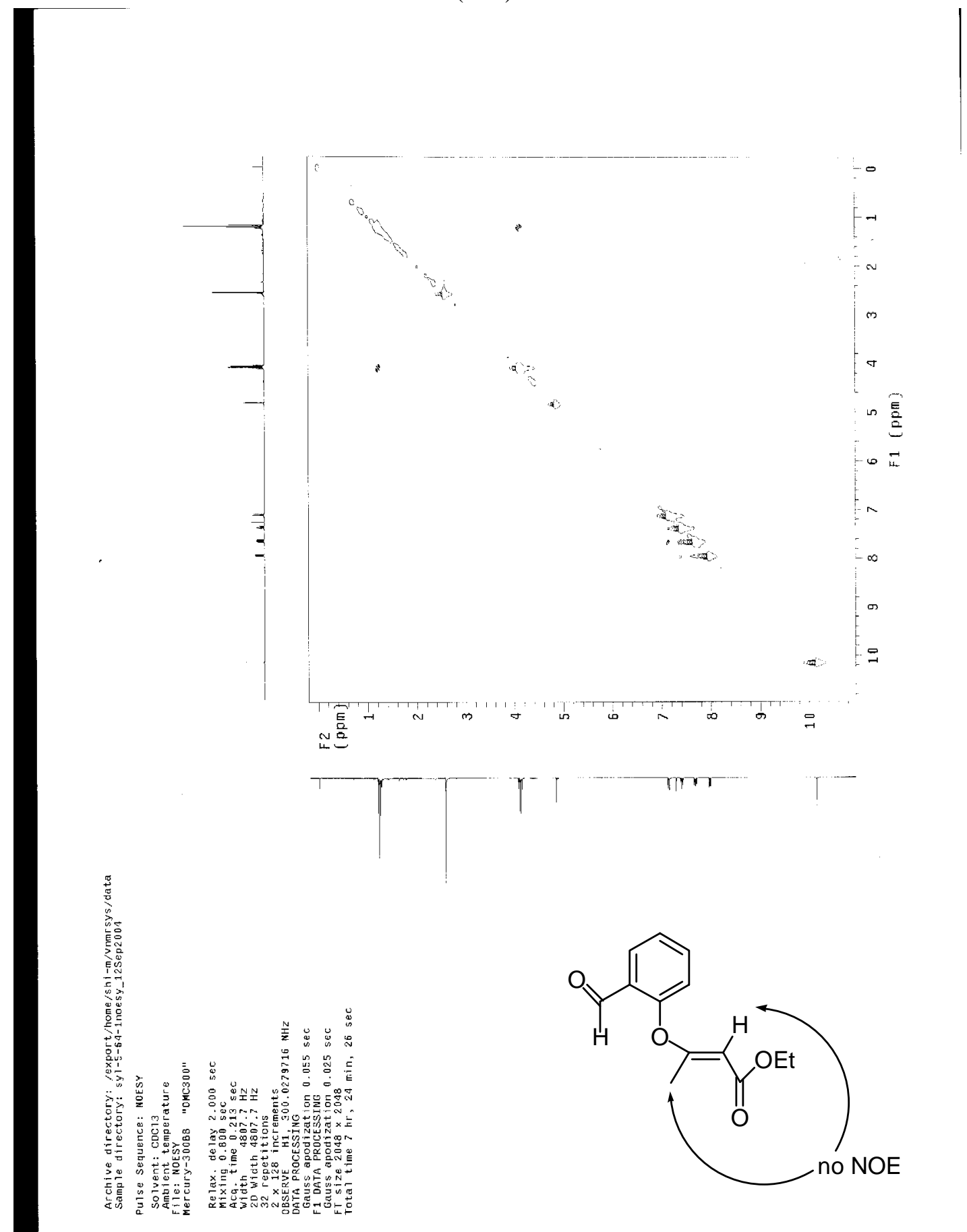

